\title{
A Numerical Exploration of Parameter Dependence in Power Optimal Flapping Flight
}

\author{
David J. Willis* Jaime Peraire ${ }^{\dagger}$ Mark Drela ${ }^{\dagger}$ Jacob K. White ${ }^{\ddagger}$ \\ Massachusetts Institute Of Technology, 77 Massachusetts Ave., Cambridge, MA 02139, USA.
}

A computational framework for analyzing and designing efficient flapping flight vehicles is presented. Two computational tools are considered: a Betz Criterion code proposed by Hall et. al., and an accelerated, unsteady, potential flow solver. The parameters considered in this paper are: the flapping frequency, the flapping amplitude in both up-down and forward-aft directions, and the addition of a mid-wing hinge for articulated flapping flight. The flapping kinematics are represented using harmonics.

Three numerical experiments are examined for the flapping flight analysis. The first experiment involves sweeping through a basic flapping flight parametric design space. The second experiment minimizes flight power at a given flight condition using a quasi-Newton optimization. The third experiment demonstrates the conversion of the problem from a wake only analysis to a 3-D flapping wing geometry.

\section{Nomenclature}

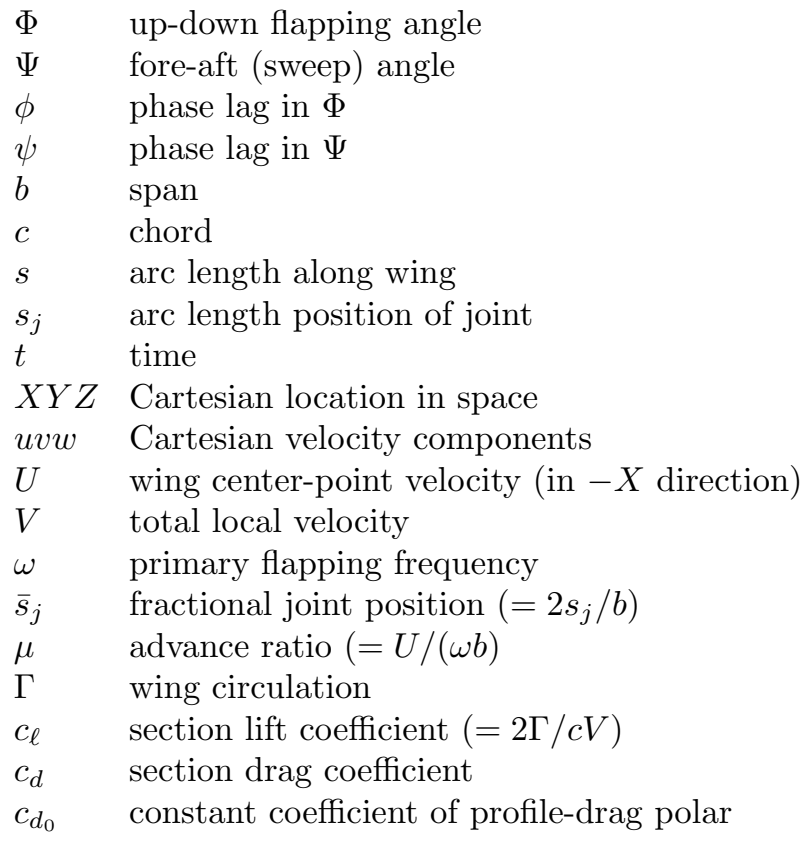

*Graduate Student, Department of Aeronautics and Astronautics, 77 Massachusetts Ave., Cambridge, MA 02139.

${ }^{\dagger}$ Professor, Department of Aeronautics and Astronautics, 77 Massachusetts Ave., Cambridge, MA 02139.

${ }^{\ddagger}$ Professor, Department of Electrical Engineering and Computer Science, 77 Massachusetts Ave., Cambridge, MA 02139. 


$\begin{array}{ll}c_{d_{2}} & \text { quadratic coefficient of profile-drag polar } \\ c_{\ell_{0}} & \text { minimum-drag } c_{\ell} \text { of profile-drag polar } \\ \rho & \text { fluid density } \\ L & \text { overall lift in } Y \text { direction } \\ T & \text { overall thrust in }-X \text { direction } \\ P & \text { overall power } \\ C_{L} & \text { overall lift coefficient }\left(=2 L / \rho U^{2} b^{2}\right) \\ C_{T} & \text { overall thrust coefficient }\left(=2 T / \rho U^{2} b^{2}\right) \\ C_{P} & \text { overall power coefficient }\left(=2 P / \rho U^{3} b^{2}\right)\end{array}$

\section{Introduction}

This paper investigates flapping flight using several low fidelity, highly efficient, computational tools. From an engineering perspective, understanding wing kinematic parametric dependence can guide design of more effective flight vehicles. From a biological perspective, understanding the importance of parametric dependencies can provide insight into natural flight kinematics as well as the evolutionary process resulting in flight. There have been several presentations of flapping flight parameters. ${ }^{1,2,4,5,8,13}$ This paper makes use of computational approaches which are well suited to understand the basic parameter dependencies while utilizing moderate computational resources.

Due to the complexity of flapping flight kinematics, Euler and Navier Stokes solvers ${ }^{6,7}$ are not considered practical for parameter dependence investigations, and lower fidelity tools are therefore used here. Extensive use will be made of the large flapping amplitude wake-only-Betz criterion methods presented by Hall et $a l .{ }^{9-11}$ The Hall et. al. approach considers a prescribed wake shape, defined by a specified harmonic motion of the generating lifting line. A set of force constraints are then applied to determine the minimum-power vorticity distribution on this wake. A vortex-lattice method is used to relate the wake's velocity field to the vorticity distribution. In the extended method in Hall et al., ${ }^{11}$ a viscous profile drag and stall model are included in the optimization. In this work a more general wake geometry description is used, with a mid-span joint and higher-harmonic motion being allowed. As in Hall, ${ }^{11}$ a simple quadratic-polar profile drag model is included.

In this paper we also demonstrate the use of an accelerated, unsteady potential flow panel method ${ }^{12}$ for analyzing the wakes which result from the Hall et al. approach. This panel method has features which are particularly effective in flapping flight analysis: a rapid solution of a morphing body problem due to the advanced acceleration algorithms, ${ }^{21,22,28}$ an automatic wake generation procedure using vortex particle wakes ${ }^{23-25}$ and the ability to model both thin-membrane and thick body representations of the geometry.

The flight parameters of interest in the current investigation are motivated by observations of bird flight: the wing flapping frequency, ${ }^{4,11}$ flapping amplitude, ${ }^{11,15}$ forward-aft wing motion, ${ }^{13,18}$ a second flapping harmonic, ${ }^{13,18}$ and a mid-wing joint (articulated flapping).

In order to demonstrate the versatility of the computational framework, three experiments are considered. The first experiment involves sweeping through the basic flapping flight parametric design space. The second experiment demonstrates a quasi-Newton optimization to minimize flight power. The third experiment demonstrates the conversion of the problem from a wake only Betz analysis to a 3-D flapping wing geometry.

\section{The Geometric and Kinematic Parameters of Interest}

The following parameters of symmetric flapping flight are considered in the present investigation.

1. Flapping Frequency (or advance ratio of Strouhal number): Several researchers have examined flapping frequency in both experimental settings, ${ }^{5,15}$ and in computational settings. ${ }^{4,11,14}$ Hall et. al. 
presented results in their original work showing the effect of flapping frequency on simple harmonic hinged flapping wings.

2. Flapping Amplitudes: For simple hinged flapping Hall et al. ${ }^{8}$ computed the dependencies of flapping amplitude. Flapping amplitude has been examined experimentally and presented for both birds ${ }^{13}$ and bats. ${ }^{13,15}$

3. Flapping with a Second Harmonic: Birds and bats demonstrate a non-unity upstroke to downstroke ratio, ${ }^{18}$ which is represented here using a second flapping harmonic. There is typically a bias to spending more time during the downstroke portion of flapping than the upstroke. ${ }^{13,18}$

4. Forward-Aft Flapping Kinematics: The path traveled by the wingtip is rarely a simple up-down path. ${ }^{13,18}$ The downstroke typically involves downward-and-forward motions, while the upstroke will in most cases involve upward-and-rearward motions.

5. Multiply Hinged Wings: In nature the existence of articulated wings is prevalent. Observing bird flight, the wing is fully extended during the downstroke and will fold during the upstroke. The degree of folding during the upstroke can be significant in certain flight regimes. ${ }^{13}$ Similarly, bats will undergo a nearly fully extended wing downstroke, with a wing-folding of some proportion during the upstroke. ${ }^{13}$

Natural flapping flight is often used as a model for flapping flight; however, it is unclear whether the features of natural flapping flight have evolved primarily to minimize flight power, or whether other survival considerations such as maneuverability or skeletal or muscular limitations have also played a significant role. Understanding these trade offs is a primary goal of the present work.

\section{A. The Wing Geometry Kinematics}

The wake geometry is defined as the trace of a two-segment lifting line representing the wing, illustrated in figure 1.

The segment orientations are defined by the flap angles $\Phi_{i}$ and $\Phi_{o}$, and the two sweep angles $\Psi_{i}$ and $\Psi_{o}$. The time variation of these angles is assumed to be the sum of two harmonics of frequency $\omega$ and $2 \omega$, with various appropriate phase angles $\phi$ and $\psi$ included.

$$
\begin{aligned}
\Phi_{i}(t)=\Phi_{i, 0} & +\Phi_{i, 1} \cos (\omega t) \\
& +\Phi_{i, 2} \cos \left(2 \omega t+\phi_{i, 21}\right) \\
\Phi_{o}(t)=\Phi_{o, 0} & +\Phi_{o, 1} \cos \left(\omega t+\phi_{o, i}\right) \\
& +\Phi_{o, 2} \cos \left(2 \omega t+\phi_{o, i}+\phi_{o, 21}\right) \\
\Psi_{i}(t)=\Psi_{i, 0} & +\Psi_{i, 1} \cos \left(\omega t+\psi_{\phi}\right) \\
& +\Psi_{i, 2} \cos \left(2 \omega t+\psi_{\phi}+\psi_{i, 21}\right) \\
\Psi_{o}(t)=\Psi_{o, 0} & +\Psi_{o, 1} \cos \left(\omega t+\psi_{\phi}+\psi_{o, i}\right) \\
& +\Psi_{o, 2} \cos \left(2 \omega t+\psi_{\phi}+\psi_{o, i}+\psi_{o, 21}\right)
\end{aligned}
$$

The position of a point $(X, Y, Z)$ on the lifting line, at arc length position $s$ and time $t$, is then given as follows.

$$
X(s, t)= \begin{cases}-U t+s \cos \Phi_{i}(t) \sin \Psi_{i}(t) & , \quad 0 \leq s \leq s_{j} \\ -U t+s_{j} \cos \Phi_{i}(t) \sin \Psi_{i}(t)+\left(s-s_{j}\right) \cos \Phi_{o}(t) \sin \Psi_{o}(t) & , \quad s_{j} \leq s \leq b / 2\end{cases}
$$




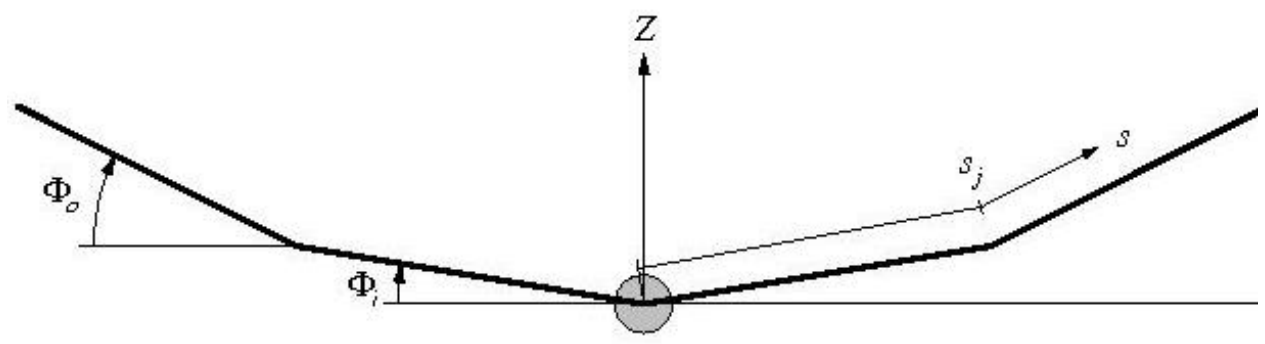

a) Rear view

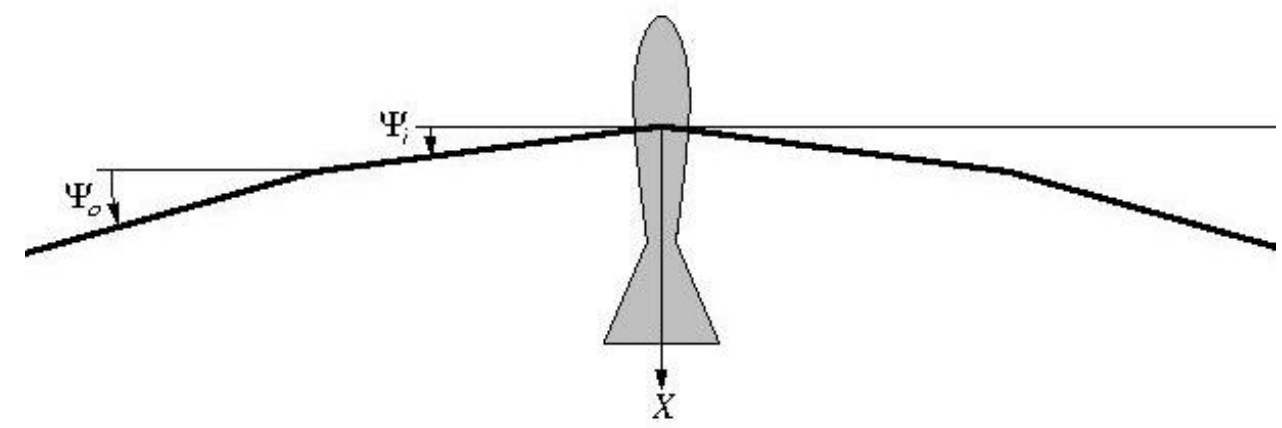

Figure 1. Lifting-line wing geometry, and flapping and sweep angles.

$$
\begin{aligned}
& Y(s, t)= \begin{cases}s \cos \Phi_{i}(t) \cos \Psi_{i}(t) & 0 \leq s \leq s_{j} \\
s_{j} \cos \Phi_{i}(t) \cos \Psi_{i}(t)+\left(s-s_{j}\right) \cos \Phi_{o}(t) \cos \Psi_{o}(t) & , \quad s_{j} \leq s \leq b / 2\end{cases} \\
& Z(s, t)= \begin{cases}s \sin \Phi_{i}(t) & , \quad 0 \leq s \leq s_{j} \\
s_{j} \sin \Phi_{i}(t)+\left(s-s_{j}\right) \sin \Phi_{o}(t) & , \quad s_{j} \leq s \leq b / 2\end{cases}
\end{aligned}
$$

The velocity components of the point on the wing relative to the fluid are determined by taking the derivatives of the above expressions with respect to $t$.

$$
\begin{aligned}
& u(s, t)=\frac{\partial X}{\partial t} \\
& v(s, t)=\frac{\partial Y}{\partial t} \\
& w(s, t)=\frac{\partial Z}{\partial t} \\
& V(s, t)=\sqrt{u^{2}+v^{2}+w^{2}}
\end{aligned}
$$

For brevity, the resulting derivative expressions are not given here. The total velocity $V$ of the wing relative to the fluid is needed to obtain the local lift coefficient in terms of the circulation, which is in turn used to define the drag coefficient for the viscous drag model.

The location of any point on the wake is specified by its $s, t$ coordinates in the $X, Y, Z$ functions above, where $t$ is the (negative) time in the past when that point was shed. The overall wake shape is therefore

$$
4 \text { of } 31
$$


specified by the following 19 parameters.

$$
\begin{aligned}
& \left\{\begin{array}{llllll}
\Phi_{i, 0} & \Phi_{i, 1} & \Phi_{i, 2} & \Phi_{o, 0} & \Phi_{o, 1} & \Phi_{o, 2}
\end{array}\right. \\
& \Psi_{i, 0} \quad \Psi_{i, 1} \quad \Psi_{i, 2} \quad \Psi_{o, 0} \quad \Psi_{o, 1} \quad \Psi_{o, 2} \\
& \begin{array}{llllll}
\phi_{i, 21} & \phi_{o, i} & \psi_{\phi} & \psi_{i, 21} & \psi_{o, i}
\end{array} \\
& \left.\bar{s}_{j} \mu\right\}
\end{aligned}
$$

Figure 2 shows several wake traces generated for a selection of these parameters. This illustrates the wide variety of flapping motions and corresponding wake shapes which can be defined by this parameterization.

\section{A Brief Description of the Simulation Tools}

The two approaches which are used in this work are discussed briefly in this section. The details pertaining to the implementation and theory of each of the methods can be found in the respective references. ${ }^{9-12}$

\section{A. The Hall et al Method}

The Hall et. al. approach ${ }^{9-11}$ was implemented in a form similar to that presented in the references. The Hall et al. method determines the circulation distribution on prescribed periodic wake shape which minimizes the total time-average power required to achieve specified average forces. The total power includes the viscous power, which is computed using a quadratic profile drag polar using the lift coefficient determined from the instantaneous circulation.

$$
\begin{aligned}
c_{\ell} & =\frac{2 \Gamma}{c V} \\
c_{d}\left(c_{\ell}\right) & =c_{d_{0}}+c_{d_{2}}\left(c_{\ell}-c_{\ell_{0}}\right)^{2}
\end{aligned}
$$

By augmenting the system using Lagrange multipliers, the total power can be minimized with respect to a set of prescribed inviscid resultant forces. The solution process is described as follows:

1. Prescribe a vortex-lattice wake geometry representation based on the trace of the wing trailing edge. The wing trailing edge trace is determined using the flapping parameters outlined earlier.

2. Prescribe the force constraints for the particular geometry.

3. Prescribe the viscous model parameters $\left(c, c_{\ell_{0}}, c_{d_{0}}, c_{d_{2}}\right)$.

4. Solve for the circulation and corresponding vorticity distribution which minimizes the power.

The approach of Hall et al. requires only that the wake trace be specified, and hence is a fairly powerful tool for flapping parameter exploration. However, since the flapping wing geometry is only minimally accounted for, it is unclear how effectively the optimum vorticity distribution can be generated by a realizable wing. Given a wing with infinite actuation possibilities, clearly the generation of the optimum wake vorticity distribution is an easy task, however, in most practical situations geometric constraints are present which prevent the production of an optimal wake. As a result, the authors have performed some preliminary coupling of the Hall wake only method with an unsteady potential flow solver to investigate the wing geometry necessary to generate the power optimal wake vorticity distribution.

\section{B. The Panel Method Simulation Methodology}

The unsteady panel method used in this work is FastAero. ${ }^{12}$ The FastAero approach has the following features which are particularly advantageous to flapping flight analysis:

5 of 31

American Institute of Aeronautics and Astronautics 
1. Vortex Particle Representation of the wake: The unsteady motion of the wake is represented using a vortex particle method. ${ }^{23-26}$ Since the vortex particles advect freely with the local velocity, and do not require connectivity data in their representation, they are particularly well suited for the automatic wake generation problem.

2. Accelerated solution methods: The use of a precorrected-FFT ${ }^{28}$ matrix vector product accelerated GMRES $^{27}$ iterative solution technique, the scalar potential flow problem is rapidly solved. In addition, a Fast Multipole Tree ${ }^{21,22}$ acceleration routine is used to accelerate the vortex particle component of the solution method. The combined p-FFT-FMT approach provides the necessary acceleration allowing morphing body problems to be computed rapidly and accurately.

3. Membrane and thick body formulations: FastAero has several boundary integral formulations implemented in the solution framework. Therefore, it is possible to represent the wing as an infinitely-thin prescribed geometry wing, or as a wing with a prescribed thickness profile. The advantage of a thin body representation is that the grid generation for morphing bodies is significantly easier than the thick body mesh generation problem. The thick body formulation is useful for detailed analysis of flapping flight when the airfoil profile is relevant.

Although FastAero has several advantages for flapping flight analysis, the method does not include a viscous correction. Viscous effects can be approximated using the section local velocity, the section bound circulation, and a drag polar for the airfoil under consideration at the Reynolds number desired; however, future plans include the incorporation of a viscous model into the solution framework.

\section{Numerical Experiments}

Several numerical experiments are considered in this paper demonstrating the versatility of the computational framework which has been implemented.

\section{A. Numerical Experiment 1}

The first numerical experiment considers a parametric design space sweep using an implementation of the Hall et. al. approach. Due to the rapid computation of the minimum power wake vorticity distribution, design space sweeps with a small number of parameters are possible. However, limitations on the number of parameters are strict due to the exponential increase in computational complexity. Practical moderate fidelity design space sweeps are limited to computations with 4-5 variables. The following pseudo code illustrates the parameter sweep process:

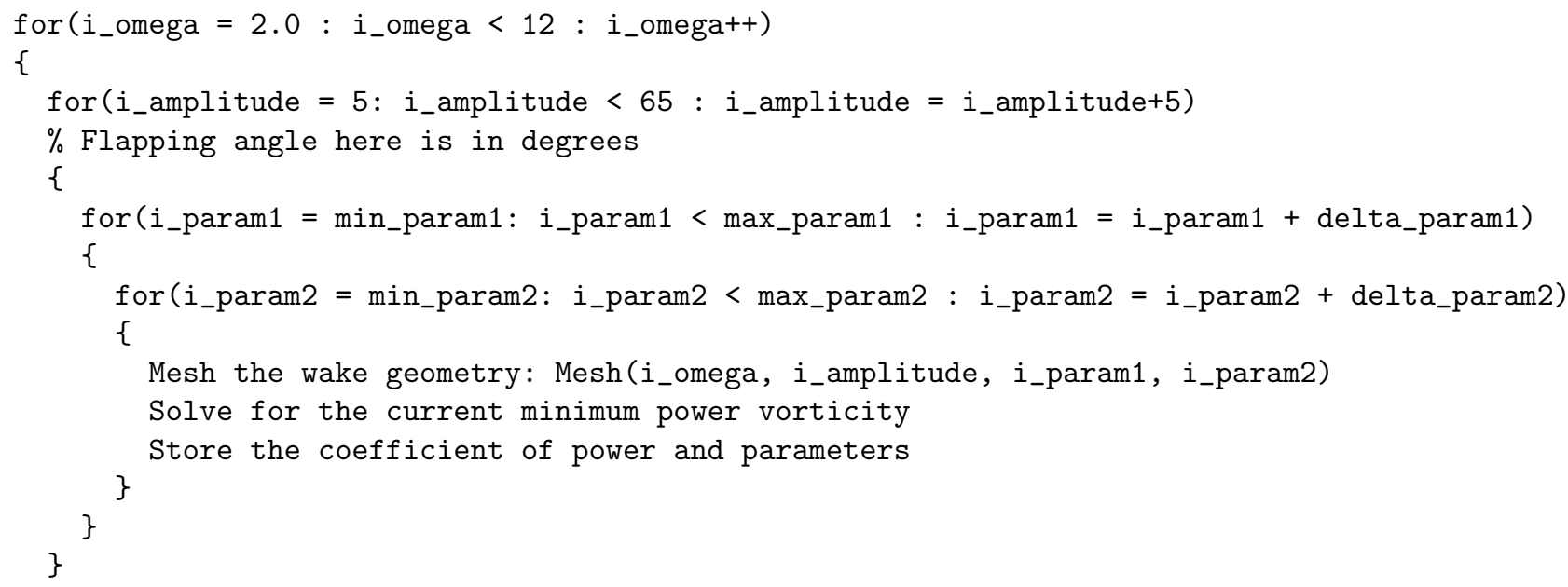


Results of the design space sweeps are presented based on the minimum power for a given pair of advance ratio and inner-first harmonic up-down flapping angle values. This is considered in order to compare the resulting minimum power coefficient to a simple flapping wing baseline model consisting only of first harmonic up-down flapping motions and a variable advance ratio. Although minimum power flight is desirable, equally important is the sensitivity of that minimum power flight to off-design operation.

\section{Flapping Frequency and Amplitude}

In the original work by Hall et. al., they investigate the effect of flapping frequency and amplitude. For comparison, we perform a similar investigation using the following parameter values:

$$
\begin{aligned}
& \mu^{-1}=2,3 \ldots 10 . \\
& \Phi_{o, 0}=\Phi_{i, 0}=\Phi_{o, 2}=\Phi_{i, 2}=0 \\
& \Phi_{o, 1}=\Phi_{i, 1}=\frac{\pi}{36}, 2 \frac{\pi}{36} \ldots 13 \frac{\pi}{36} .
\end{aligned}
$$

Figure 3 illustrates the power coefficient variation with the flapping advance ratio and the flapping amplitude for several different flight conditions.

\section{Discussion}

The simple hinged flapper demonstrates several features of efficient flapping flight. As noted by Hall et al. ${ }^{11}$ there is a low sensitivity of minimum power flight to advance ratio across a large interval of practical values. This low sensitivity is due to the ability to tune the flapping amplitude at a given non-optimal advance ratio such that the overall power is minimized. This tuning of the flapping amplitude enables efficient and effective operation across a wide range of velocities, flapping frequencies and flapping amplitudes. Furthermore, the low sensitivity of the power coefficient to sub-optimal parameter choices in both flapping amplitude and advance ratio provides low off design flight power consumption.

In the sections which follow, comparisons of this baseline hinged flapping flight model are made for situations in which additional flapping degrees of freedom are introduced.

\section{Forward-Aft Wing Stroke Behavior}

In this example the fore-aft wing flapping are considered, in addition to the up-down motion. The phase between the up-down and fore-aft motion is also considered. The design space sweeps are performed over:

$$
\begin{aligned}
& \mu^{-1}=2,3 \ldots 10 \\
& \Phi_{o, 0}=\Phi_{i, 0}=\Phi_{o, 2}=\Phi_{i, 2}=0 \\
& \Phi_{o, 1}=\Phi_{i, 1}=\frac{\pi}{36}, 2 \frac{\pi}{36} \ldots 13 \frac{\pi}{36} \\
& \Psi_{o, 0}=\Psi_{i, 0}=\Psi_{o, 2}=\Psi_{i, 2}=0 \\
& \Psi_{o, 1}=\Psi_{i, 1}=\frac{\pi}{36}, 2 \frac{\pi}{36} \ldots 6 \frac{\pi}{36} \\
& \psi_{o, 21}=\psi_{i, 21}=\frac{\pi}{8}, \frac{\pi}{4} \ldots 2 \pi
\end{aligned}
$$

The results are shown in figure 4. The wake shape corresponding to the minimum power solution in the design space sweep is illustrated in figures 5-6.

\section{Discussion}

The design space sweep over an interval of forward-aft flapping angles demonstrates the following:

1. The additional degree of freedom associated with the forward-aft motion results in lower power consumption. However, it should be noted that the gains are small for low thrust situations and may not offset the cost associated with prescribing the motion. 
2. The forward-aft motion which minimizes power matches well with the observed behavior in natural flapping flight. Although it is natural for wing-flapping animals to have their wings advance during the downstroke (due to the production of thrust over the wings), it may also serve to reduce their power consumption.

3. At higher thrust to lift ratios, the forward-aft flapping motion becomes more effective in reducing power consumption. The coupled forward-aft, up-down motion of the wings results in a wake whose downstroke trace covers a large area and lies in a plane which is close to perpendicular to the desired resultant force. The forward-aft motion of the wings can therefore make the downstroke more closely resemble a tilted actuator disk. Based on these results, increased forward aft motion of the wings is therefore expected in steep climbing flight, slow flight, and during takeoff. The resultant of the lift and thrust effectively act together to produce motion in these flight regimes.

4. The motion which results in optimal minimum power flight implies forward-downward motion of the wing during the downstroke and upward-rearward motion during the upstroke. The corresponding wake shapes are illustrated in figures 5-6.

5. As the advance ratio tends toward zero, the amplitude of the forward-aft motions are reduced to achieve minimum power consumption. This effect is likely seen in the computations in order to reduce viscous power loss at higher reduced frequencies. In addition, as the up-down flapping amplitude increases, the forward-aft wing motions are attenuated. Again, this is likely a result of curbing the viscous power loss associated with more rapid relative motions.

\section{The Second Harmonic}

In this numerical experiment the addition of a second harmonic to the wing kinematics is examined. In this simulation the design space sweep is performed over:

$$
\begin{aligned}
& \mu^{-1}=2,3 \ldots 10 \\
& \Phi_{o, 0}=\Phi_{i, 0}=0 \\
& \Phi_{o, 1}=\Phi_{i, 1}=\frac{\pi}{36}, 2 \frac{\pi}{36} \ldots 13 \frac{\pi}{36} \\
& \Phi_{o, 2}=\Phi_{i, 2}=\frac{\pi}{36}, 2 \frac{\pi}{36} \ldots 13 \frac{\pi}{36} \\
& \phi_{o, 21}=\phi_{i, 21}=\frac{\pi}{8}, \frac{\pi}{4} \ldots 2 \pi
\end{aligned}
$$

Figures $7-8$ illustrate the second harmonic design space search. Figures 9-10 illustrate the wake shapes corresponding to the minimum power coefficient determined during the design space sweep.

\section{Discussion}

The results of the design space sweep appear to demonstrate a greater positive effect on power reduction than is actually observed. This is due to the method chosen for representing the results. When the flapping amplitude of the first harmonic is low, the second harmonic assumes the role of the dominant flapping amplitude. Therefore, the apparent reduction in power which is illustrated in the figures is not as significant as it appears. This is a pronounced effect in the figures 7 and 8 when $\Phi_{o, 1}<0.3$ radians.

The minimum power required for flapping when considering both the first and second harmonics is less than the minimum power of the baseline result. Considering two harmonics enables the downstroke wake to adopt a more effective shape in for producing the flight forces.

\section{Articulation Result Behavior}

The final parameter which is considered in the design space sweep experiment is the mid-wing joint (or articulation) of the wing. In this simulation the design space sweep is performed over: 


$$
\begin{aligned}
& \mu^{-1}=2,3 \ldots 10 . \\
& \Phi_{o, 0}=\Phi_{i, 0}=\Phi_{o, 2}=\Phi_{i, 2}=0 \\
& \Phi_{i, 1}=\frac{\pi}{36}, 2 \frac{\pi}{36} \ldots 13 \frac{\pi}{36} \\
& \Phi_{o, 1}=\frac{\pi}{36}, 2 \frac{\pi}{36} \ldots 13 \frac{\pi}{36} \\
& \phi_{i, o}=\frac{\pi}{8}, 2 \frac{\pi}{8} \ldots 2 \pi
\end{aligned}
$$

The results of the Articulation design space sweep are illustrated in figures $11-12$. Figures 13-14 illustrate the wake shapes corresponding to the minimum power coefficient determined during the design space sweep for articulated flapping flight.

\section{Discussion}

Considering the results in figure 11, articulated flapping flight tends to reduce the minimum power while also enlarging the region of minimum power. As a result the minimum power solution is less sensitive to the flapping parameters than the baseline model. Comparing figures 11 and 12 to the baseline example demonstrates the advantage of articulation when the flapping amplitude increases and the advance ratio decreases. This suggests that the viscous power loss is minimized in the articulated flapping flight simulations. In fact, in the current model articulation results in lower relative velocities at the wing tips therefore minimizing the viscous power loss. This viscous power savings effect is more pronounced when large flapping amplitudes are considered.

In nature many birds fold their wings to a certain extent during the upstroke of the wingbeat. One significant reason for an articulated upstroke is the ease in which the outer region of the wing can be unloaded during the upstroke. If birds did not have an articulated wing, significant twist would be required to have a lightly loaded wingtip region during the upstroke motion. The Hall et al. model does not constrain the wing twist required to create the optimal vorticity distribution. Therefore, it is likely that articulation serves the dual purpose of providing a functional morphology resulting in a lightly loaded upstroke motion while also providing a means for overall flight power reduction.

\section{B. Numerical Experiment 2: Optimization of the Flight Power}

In the second numerical experiment, a more rigorous approach for minimizing flapping flight power is considered. The flight power coefficient is minimized for specified flight conditions over a restricted set of flapping parameters using a BFGS ${ }^{19}$ quasi-Newton method. ${ }^{20}$ A $3 \times 2$ array of flight parameters is examined for the flapping problem. Three different values of $c_{d_{0}}=c_{d_{2}}=0.01,0.03,0.10$ are considered for two separate approximate climb angles $\left(\gamma_{\text {approx }}=10^{\circ}, 30^{\circ}\right)$. The goal of this experiment was to determine the optimal set of parameters for a given set of flight conditions.

\section{Setting up the Quasi-Newton Method}

The following pseudo code demonstrates the quasi-Newton approach taken in this experiment:

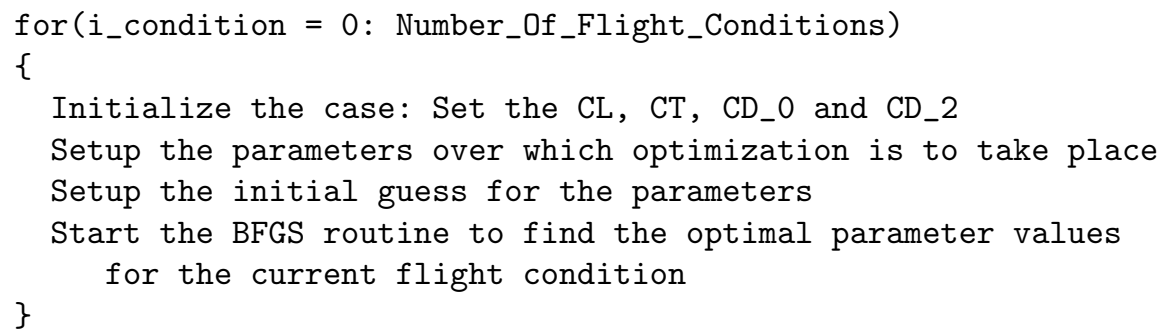

The gradients used in the BFGS optimization are computed using a finite difference approach, hence, as the number of parameters increases, the cost associated with the gradient evaluations increases. In future implementations an adjoint based approach for sensitivity computations will be considered. 


\section{A Note Regarding Solution Optimality}

It should be noted that the results presented in this section represent local minimums. Although an effort was made to explore different initial conditions in the design space, the power dependence on flapping parameters in flapping flight has many local minimums. As such, we present the optimal results for the search for minimum power. In addition, the design space over which the optimization is taking place presents a challenge to gradient based approaches due to the low sensitivity of the minimum power to the flapping frequency in large regions of the space.

In this experiment, the design space under consideration has six free parameters over which the optimization process is carried out.

\section{Baseline Simple Hinged Flapping}

The simple hinged flapping minimum power results for several different flight conditions are presented in table 1. In this case the advance ratio, and the maximum and minimum up-down flapping angles were set as parameters in the optimization environment.

\begin{tabular}{|r|r|r|r|r|r|r|}
\hline Parameter & Case 1r & Case 2r & Case 3r & Case 4r & Case 5r & Case 6r \\
\hline$C_{L}$ & 0.10 & 0.10 & 0.10 & 0.10 & 0.10 & 0.10 \\
$C_{T}$ & 0.058 & 0.058 & 0.058 & 0.018 & 0.018 & 0.018 \\
$c_{d_{0}}$ & 0.01 & 0.03 & 0.10 & 0.01 & 0.03 & 0.1 \\
$\mu^{-1}$ & 5.55 & 5.54 & 5.53 & 5.51 & 5.50 & 5.47 \\
$\Phi_{o, 1}$ & 0.79 & 0.69 & 0.58 & 0.46 & 0.39 & 0.37 \\
\hline$C_{P}$ & 0.01687 & 0.02692 & 0.05821 & 0.008176 & 0.01392 & 0.03297 \\
\hline
\end{tabular}

Table 1. The optimal values found for the baseline simple flapping flight with different $c_{d_{0}}$, when the climb slope is near $30^{\circ}$, and $10^{\circ}$.

\section{Forward-Aft Wing Stroke Behavior}

The effect of allowing forward-aft flapping motions is investigated. Results are presented in Table 2.

\section{Discussion}

The results of the coefficient of power minimization presented in Table 2 demonstrate the power reduction in climbing (or increased thrust) flapping flight when a forward-aft flapping motion is combined with an up-down flapping motion. This result also tends to confirm the proposition that the downstroke motion is best coupled with forward wing motion and the upstroke motion is best coupled with aft motion of the wing when larger climb angles are prescribed. When less extreme flight conditions are considered, the benefits of forward-aft flapping motions are less apparent in the current analysis. The result from this numerical experiment are more general than those from the design space sweep due to the larger number of design parameters considered. In this example, the maximum and minimum flapping angles are not constrained to be equal, therefore, highly unsymmetrical motions can result. The reduction of power by $7 \%$ represents a significant improvement in power consumption; however, this level of power savings is only present when considering more extreme flight conditions (in this case an approximate climb angle of $30^{\circ}$ is prescribed). 


\begin{tabular}{|r|r|r|r|r|r|r|}
\hline Parameter & Case 1a & Case 2a & Case 3a & Case 4a & Case 5a & Case 6a \\
\hline$C_{L}$ & 0.10 & 0.10 & 0.10 & 0.10 & 0.10 & 0.10 \\
$C_{T}$ & 0.058 & 0.058 & 0.058 & 0.018 & 0.018 & 0.018 \\
$c_{d_{0}}$ & 0.01 & 0.03 & 0.10 & 0.01 & 0.03 & 0.1 \\
$\mu^{-1}$ & 5.48 & 4.74 & 4.76 & 5.44 & 5.38 & 5.32 \\
$\Phi_{o, 0}$ & 0.10 & 0.22 & 0.16 & 0.10 & 0.08 & 0.07 \\
$\Phi_{o, 1}$ & 0.80 & 0.76 & 0.64 & 0.46 & 0.40 & 0.35 \\
$\Psi_{o, 0}$ & 0.14 & 0.46 & -0.38 & 0.06 & 0.025 & -0.10 \\
$\Psi_{o, 1}$ & -0.39 & -0.59 & -0.39 & -0.22 & -0.12 & -0.34 \\
$\psi_{\phi}$ & -0.42 & 0.05 & 0.37 & -0.61 & -0.64 & -0.66 \\
\hline$C_{P}$ & 0.01564 & 0.02499 & 0.0550 & 0.008048 & 0.01368 & 0.03170 \\
\hline$C_{P}$ change & $-7.26 \%$ & $-7.16 \%$ & $-7.15 \%$ & $-1.56 \%$ & $-1.79 \%$ & $-3.86 \%$ \\
\hline
\end{tabular}

Table 2. The optimal values found for the forward-aft flapping motions parameter investigation with different $c_{d_{0}}$, when the climb slope is near $30^{\circ}$, and $10^{\circ}$.

\section{Articulated Wing Stroke Behavior}

The effect of allowing articulated wing flapping motions is investigated. Results are presented in Table 3.

\begin{tabular}{|r|r|r|r|r|r|r|}
\hline Parameter & Case 1b & Case $2 \mathrm{~b}$ & Case 3b & Case 4b & Case $5 \mathrm{~b}$ & Case $6 \mathrm{~b}$ \\
\hline$C_{L}$ & 0.10 & 0.10 & 0.10 & 0.10 & 0.10 & 0.10 \\
$C_{T}$ & 0.058 & 0.058 & 0.058 & 0.018 & 0.018 & 0.018 \\
$c_{d_{0}}$ & 0.01 & 0.03 & 0.10 & 0.01 & 0.03 & 0.1 \\
$\mu^{-1}$ & 5.79 & 5.54 & 5.56 & 5.53 & 5.51 & 5.51 \\
$\Phi_{i, 0}$ & 0.34 & 0.35 & 0.31 & 0.36 & 0.19 & 0.28 \\
$\Phi_{i, 1}$ & 0.58 & 0.51 & 0.45 & 0.36 & 0.28 & 0.29 \\
$\Phi_{o, 0}$ & -0.03 & -0.04 & -0.01 & -0.17 & -0.19 & -0.09 \\
$\Phi_{o, 1}$ & 0.95 & 0.80 & 0.68 & 0.39 & 0.36 & 0.31 \\
$\psi_{i o}$ & -2.19 & -2.20 & -2.15 & -2.24 & -2.14 & -2.24 \\
\hline$C_{P}$ & 0.01616 & 0.02594 & 0.0566 & 0.00803 & 0.01367 & 0.03283 \\
\hline$C_{P}$ change & $-4.16 \%$ & $-3.64 \%$ & $-2.81 \%$ & $-1.84 \%$ & $-1.84 \%$ & $-0.42 \%$ \\
\hline
\end{tabular}

Table 3. The optimal values found for articulated wing flapping motions with different $c_{d_{0}}$, when the climb slope is near $30^{\circ}$, and $10^{\circ}$.

Discussion

The effects of articulation presented in the above table demonstrate that articulated flapping flight can reduce the flight power over the baseline optimum.

\section{Discussion of Experiments 1 and 2}

The first two experiments presented demonstrate the Hall et al. method which has been implemented as an exploratory tool for analysis of the basic parameter dependencies in flapping flight. The model provides an extremely powerful tool for preliminary design and analysis of power optimal flapping flight. 
The results from the simulations presented demonstrate that the simpler models for flapping flight can capture the essence of the problem using a minimal amount of computational resources. Although it is unlikely that the results from the simulations are quantitatively similar to natural flapping flight, it is likely that the trends and qualitative results can be applied to natural flapping flight. Similar to the Trefftz plane analysis for steady fixed wing aerodynamics ${ }^{29}$ the modified wake-only Betz Criterion approach presented by Hall et. al provides significant insight into efficient flapping flight.

\section{Numerical Experiment 3}

The last numerical experiment which is performed demonstrates the the process of designing an efficient flapping wing based on the solution of the Hall et. al. approach.

\section{A. Generating a Flapping Wing Geometry From The Wake Solutions}

There are several steps involved in translating the wake only representation of the wake into a three dimensional flapping wing model:

1. Construct a reference wing platform which flaps with the correct parameter dependence while shedding the least amount of vorticity possible into the domain. The wing can be designed to have a zero vorticity shedding throughout the wingbeat by instantaneous local modifications of section camber and angle of attack. In this experiment, however, the wing is assumed to have a zero camber and the wing section is aligned with the flow at the leading edge. This assumption will produce a reference wing which has non-zero vorticity, especially in regions near the tip where incidence angles are important; however, as a first order approximation for demonstrating the translation process this is adequate.

2. Compute the downwash at lifting line positions along the wake considering only the vorticity downstream of the lifting line.

3. Compute the local section angle of attack for the entire wing beat at each spanwise section of the wing. This is computed considering the optimal vorticity distribution in the wake from the Hall et. al. simulations.

4. Adjust the reference wing platform for the local downwash effects due to the wake as well as the local sectional angle of attack. The resulting geometry will have significant twist as the wing span is traversed during the flapping motions.

5. Mesh the flapping wing as either a thick body representation (using an airfoil profile) or as a thin membrane. In this paper the thin membrane is considered due to the ease of meshing the geometry (especially near flapping joints).

\section{Example Result}

The following section demonstrates the conversion of a wake only simulation result to a FastAero flapping wing geometry which is used in a simulation of flapping flight. In figure 18(a) the optimum power wake vorticity distribution is presented. In figure 18(d) the wake trace is presented as a collection of vortex particle elements. The vortex particles demonstrate the features of the wake flow such as wake roll-up in the wingtip regions during the downstroke portion of the flapping. In figure 18(b) the wake structure is represented using vortex particles. In this figure the vortex particle strength and direction is presented to demonstrate the vorticity structure in the wake.

Discussion The geometry was constructed from the optimal vorticity wake-only solution for the given wake shape. Compared with the wake only simulation result in figure 18(a), the FastAero simulation (figures

12 of 31

American Institute of Aeronautics and Astronautics 
18(d)-18(b)) shows similarities in the main wake features such as large tip vorticity during the downstroke; however, differences exist between the two wakes such as strong tip vorticity in the potential flow model during the upstroke, and some reduction in magnitude of vorticity on the inboard section of the wing during the downstroke. These differences are attributed in part to the approximate zero-lift flapping reference wing used as a foundation for the geometry description.

\section{Conclusions}

In this paper, we have presented a computational framework capable of rapidly analyzing parametric dependencies in flapping flight. In the experiments, it was discovered that permitting additional parameters in the flapping kinematics description reduced the power required for flight. The power reduction was dominant in more extreme flight cases such as situations where steep climb angles are encountered. This is not surprising due to the competing objectives of lift and thrust production in these regimes. As a result, flapping motions which adjust the wake shape such that the fluid momentum increases during the downstroke are most effectively used to propel and lift the flapping vehicle. As such, the forward-aft wing motions during flapping flight are seen to be an effective means of adjusting the wake shape to minimize power consumption during steeper climbing flight.

In addition to basic parametric study examples, a presentation of the conversion of the wake only analysis to a potential flow solver is presented. Although the results do not illustrate a perfect match between the vortex wakes, the overall wake structure is preserved in the translation. In future investigations the conversion from a wake only analysis to a panel method representation will be modified for more accurate representation. Finally, with the ability to perform rapid parameter trade-offs using the wake only analysis of Hall et al. and subsequent analysis in the FastAero panel method environment, further studies of parameter dependence will be performed.

\section{Acknowledgments}

The authors would like to express their gratitude to the Singapore-MIT Alliance, the National Sciences Foundation and the Natural Sciences and Engineering Research Council of Canada.

\section{References}

${ }^{1}$ Ellington, C. The aerodynamics of animal flight, American Zoology, Vol 24:95-105, 1984.

${ }^{2}$ Rayner, J.M.V. A vortex theory of animal flight I. The vortex wake of a hovering animal, Journal of Fluid Mechanics, Vol 91: pp697-730, 1979.

${ }^{3}$ Rayner, J.M.V. A vortex theory of animal flight II. The forward flight of birds, Journal of Fluid Mechanics, Vol 91: pp731-763, 1979. 2000.

${ }^{4}$ Wang, J., Vortex shedding and frequency selection in flapping flight, Journal of Fluid Mechanics, vol. 410: pp 323-341,

${ }^{5}$ Spedding, G.R., The Wake of A Kestrel (Falco tinnunculus) in flapping flight, Journal of Experimental Biology,Vol 127:pp59-78, 1987.

${ }^{6}$ Mittal, R, Immersed Boundary Methods, Annual Review of Fluid Mechanics, Vol. 37: 239-261, 2005.

${ }^{7}$ Kirby, R.M., Warburton, T.c., Sherwin, S.J., Beskok, A., Karniadakis, G.E., The NeKTar Code: Dynamic Simulations without Remeshing, 2nd Int'l Symposium on Computational Technologies for Fluid/Thermal/Chemical Systems with Industrial Applications, August 1-5, 1999.

${ }^{8}$ Hall, K.C., Pigott, S.A., Power Requirements for Large-Amplitude Flapping Flight, AIAA Paper 97-0827, Presented at the 35th Aerospace Sciences Meeting and Exhibit, Reno, NV, Jan. 6-9, 1997.

${ }^{9}$ Hall, S.R., Yang, K.Y., and Hall, K.C., Helicopter Rotor Lift Distributions for Minimum Induced Power Loss, Journal of Aircraft, Vol 31, No.4, pp837-845, 1994.

${ }^{10}$ Hall, K.C., and Hall, S.R., Minimum Induced Power Requirements for Flapping Flight, Journal of Fluid Mechanics, Vol. 323, pp. 285-315, 1996.

${ }^{11}$ Hall, K.C., Pigott, S.A., Hall, S.R., Power Requirements for Large-Amplitude Flapping Flight, Journal of Aircraft, Vol35, No.3, May-June 1998

13 of 31

American Institute of Aeronautics and Astronautics 
${ }^{12}$ Willis, D.J., Peraire, J., White, J.K., A Combined pFFT-multipole tree code, unsteady panel method with vortex particle wakes,43rd AIAA Aerospace Sciences Meeting and Exhibit, AIAA 2005-0854, Reno, NV, Jan. 2005.

${ }^{13}$ Norberg, U.M., Vertebrate Flight, Mechanics, Physiology, Morphology, Ecology and Evolution, Zoophysiology, Vol. 27, Springer, 1990.

${ }^{14}$ Pennychuick, C.J., Bird Flight Performance, a practical calculation manual,University Press, Oxford, 1989.

${ }^{15}$ Bullen, R.D., and McKenzie, N.L. Scaling bat wingbeat frequency and amplitude, The journal of Experimental Biology vol 205, pp2615-2626, 2002.

${ }^{16}$ Spedding, G.R., Personal Communication, Brown University

${ }^{17}$ Hendenstrom, A., Rosen, M., and Spedding, G.R. Vortex wakes generated by robins Erathacus rubecula during free flight in a wind tunnel, Journal of The Royal Society Interface, Vol 3(7): pp 263-276, April 2006.

${ }^{18}$ Alexander, D.E.,Nature's Flyers: Birds, Insects, and the Biomechanics of Flight, The Johns Hopkins University Press, 2004.

${ }^{19}$ Fletcher, R., Practical Methods of Optimization,2nd Ed., John Wiley and Sons, 2000.

${ }^{20}$ Katanforoush, A., Unconstrained Optimization Library, math.ipm.ac.ir/scc/proglib.htm.

${ }^{21}$ L. Greengard and V. Rohklin, A Fast Algorithm for Particle Simulations, J. Comp. Phys., 73:pp325-384, 1987.

${ }^{22}$ Appel, A.A., An efficient program for many body simulations', SIAM Journal of scientific and statistical computing, vol. 16, n. 1, pp85-103, 1985.

${ }^{23}$ Voutsinas S.G., Belessis M.A., and Rados K.G., Investigation of the yawed operation of wind turbines by means of a vortex particle method. AGARD-CP-552 FDP Symposium on Aerodynamics and Aeroacoustics of Rotorcraft, Berlin, Germany, Paper 11, 1995.

${ }^{24}$ Rehbach, C., Calcul numerique d'ecoulement tridimensionels instationaires avec nappes tourillonnaires, La Recherche Aerospatiale, pp.289-298, 1977.

${ }^{25}$ G. S. Winckelmansand A. Leonard, Contributions to Vortex Particle Methods for the Computation of Three-Dimensional Incompressible Unsteady Flows, J. Comp. Phys, 109, pp 247-273, 1993.

${ }^{26}$ J. T. Beale, A. Majda, Vortex methods I: convergence in three dimensions, Math. Comput. 29 (159) 1-27, 1982.

${ }^{27}$ Saad, Y., and Schultz, M.H. A generalized minimal residual algorithm for solving nonsymmetric linear systems. SIAM Journal of Scientific and Statistical Computing, Vol 7, pp 856-869, 1986.

${ }^{28}$ J. R. Philips and J. K. White, A Precorrected-FFT Method for Electrostatic Analysis of Complicated 3-D Structures, IEEE Transactions On Computer-Aided Design of Integrated Circuits and Systems, IEEE, Vol. 16, 1997.

${ }^{29}$ Ashley, H. and Landahl, M., Aerodynamics of Wings and Bodies, Addison-Wesley, Reading, MA, 1965. 


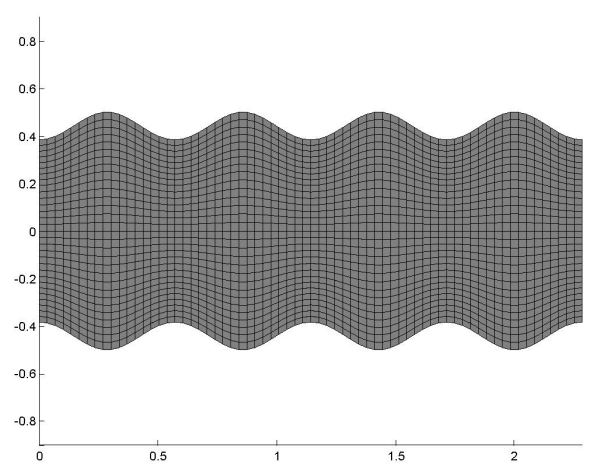

(a) Baseline simple hinged harmonic flapping

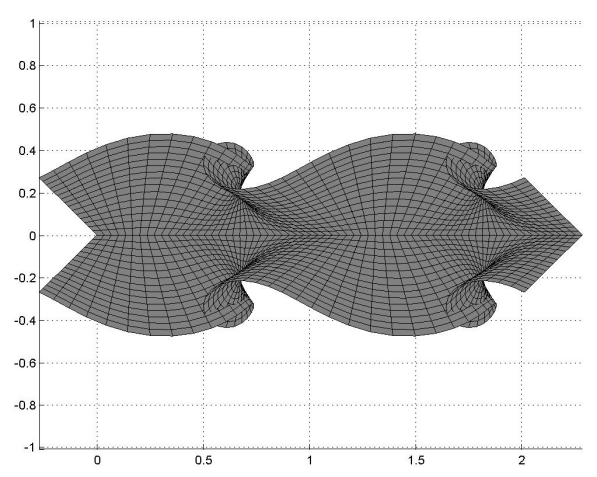

(c) Baseline + forward-aft motions.

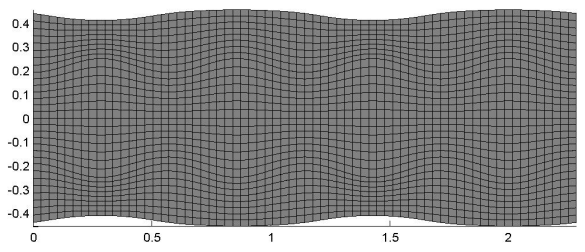

(e) Baseline + mid-wing articulation.

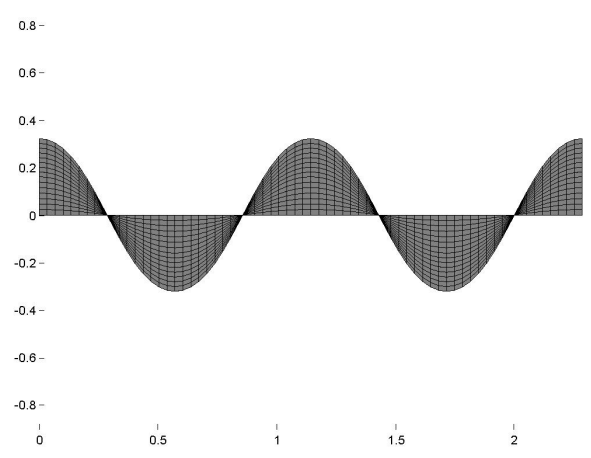

(b) Baseline simple hinged harmonic flapping.

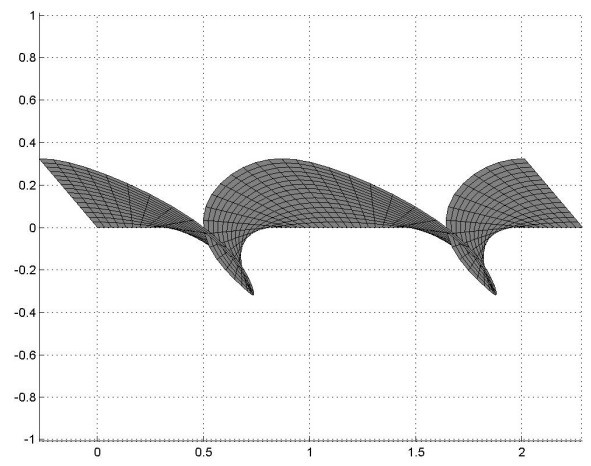

(d) Baseline + forward-aft motions.

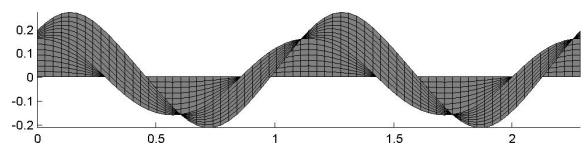

(f) Baseline + mid-wing articulation.

Figure 2. An example of the diverse wake shapes possible using the harmonic flapping kinematics model presented in this paper. Direction of travel is from left to right.

$$
15 \text { of } 31
$$




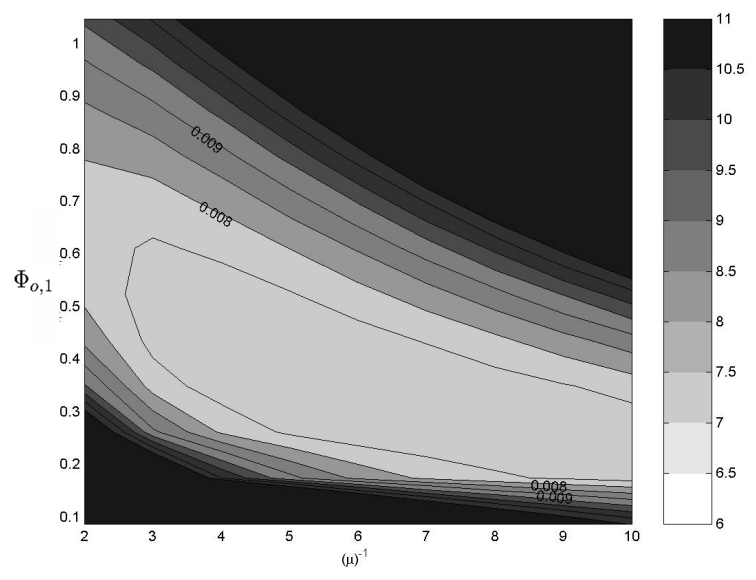

(a) $C_{T_{i}}=0.01, C_{L}=0.1, c_{d_{0}}=0.01$.

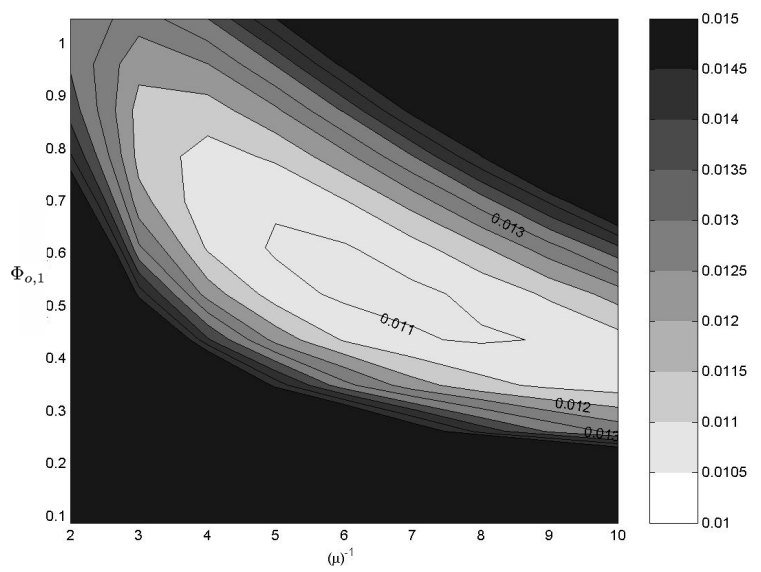

(c) $C_{T_{i}}=0.03, C_{L}=0.1, c_{d_{0}}=0.01$.

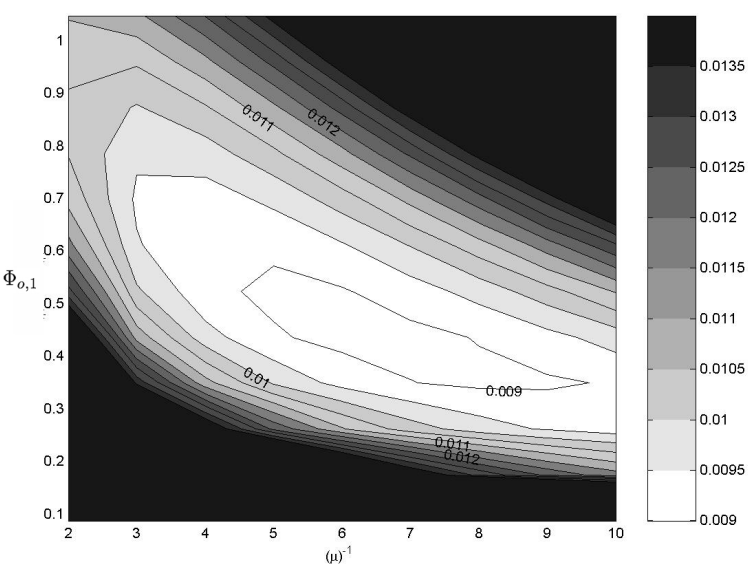

(b) $C_{T_{i}}=0.02, C_{L}=0.1, c_{d_{0}}=0.01$.

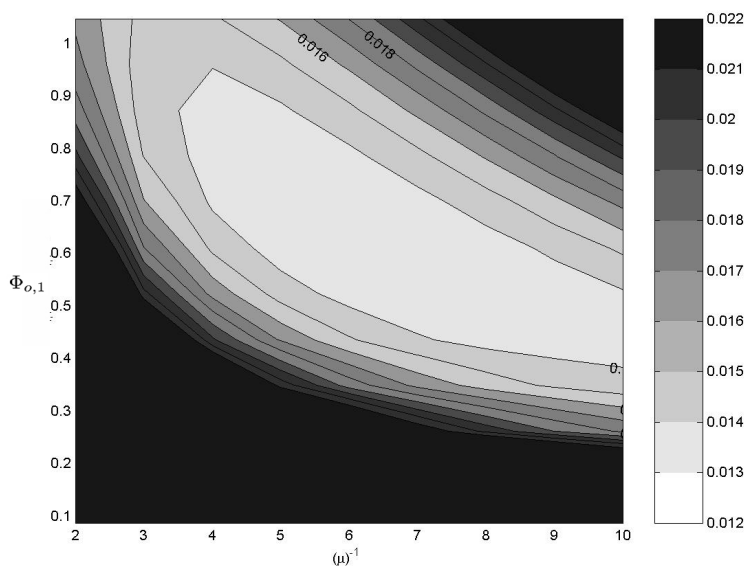

(d) $C_{T_{i}}=0.04, C_{L}=0.1, c_{d_{0}}=0.01$.

Figure 3. Power coefficient plotted as a function of the flapping amplitude and advance ratio, for $C_{T_{i}}=$ $0.01,0.02,0.03$, and $0.04, C_{L}=0.1, c_{d_{0}}=0.01$. 


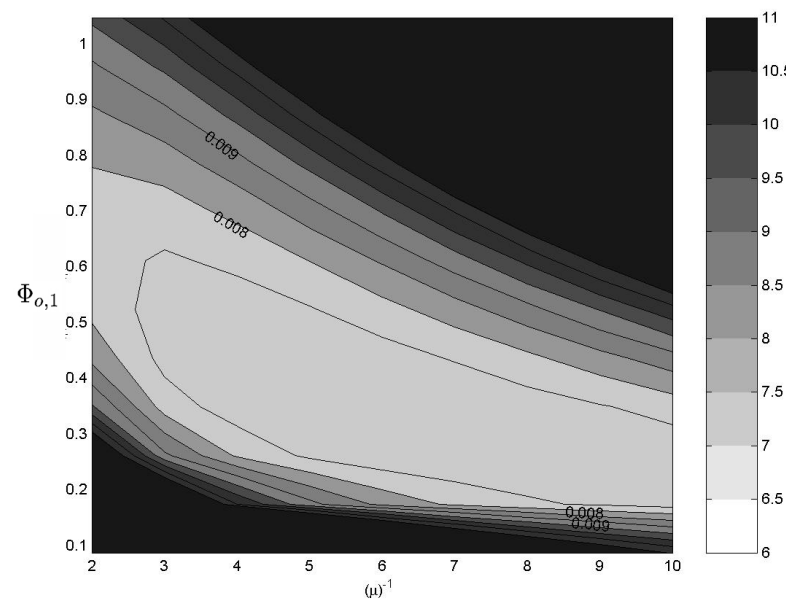

(a) Baseline, $C_{T i}=0.01$.

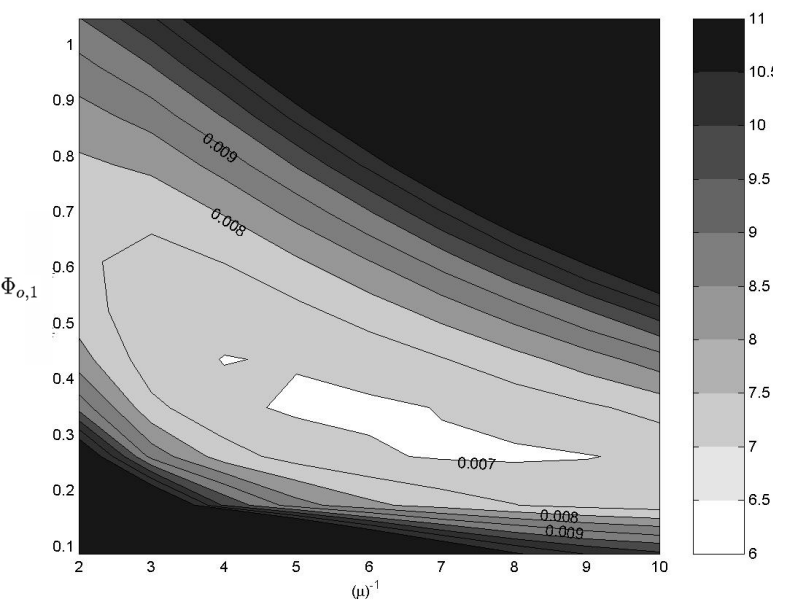

(c) Forward-Aft, $C_{T i}=0.01$.

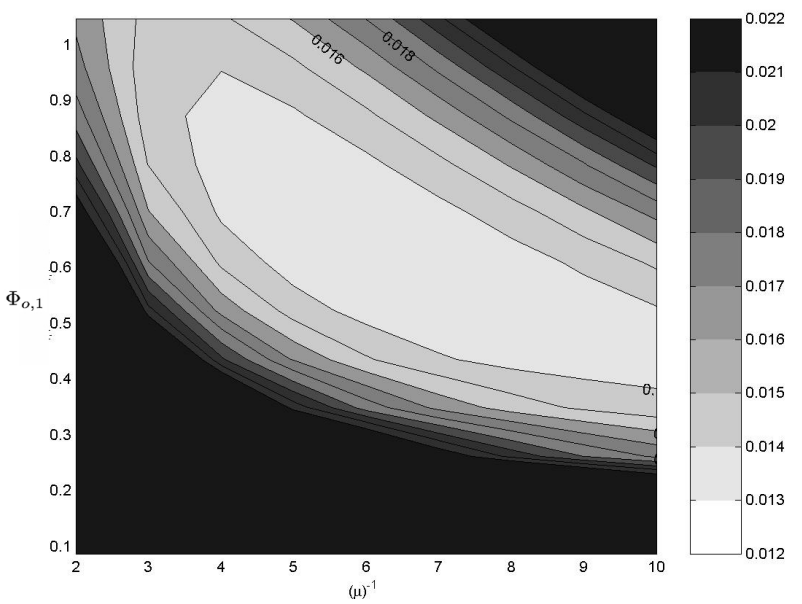

(b) Baseline, $C_{T i}=0.04$.

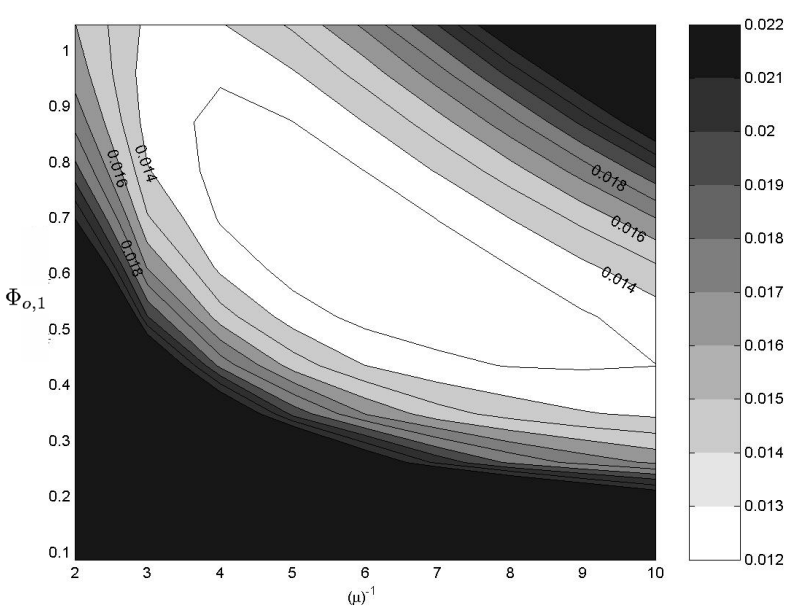

(d) Forward-Aft, $C_{T i}=0.04$.

Figure 4. An illustration of the minimum power at a given root up-down flapping amplitude and advance ratio for the Baseline (simple up-down flapping motion) with one which has a forward-aft motion included in the wing kinematics model. 


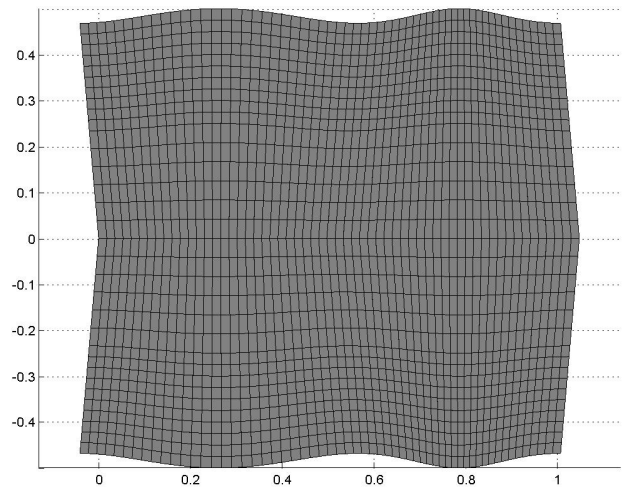

(a) Top View.

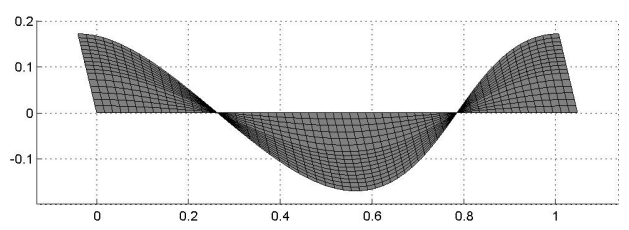

(c) Side View

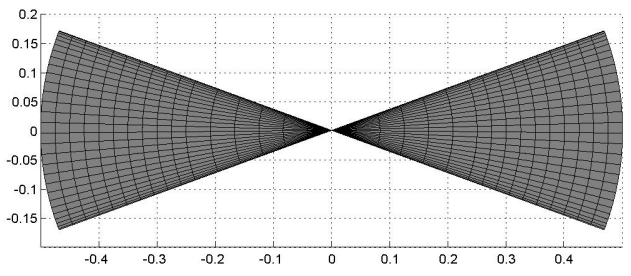

(b) Front View.

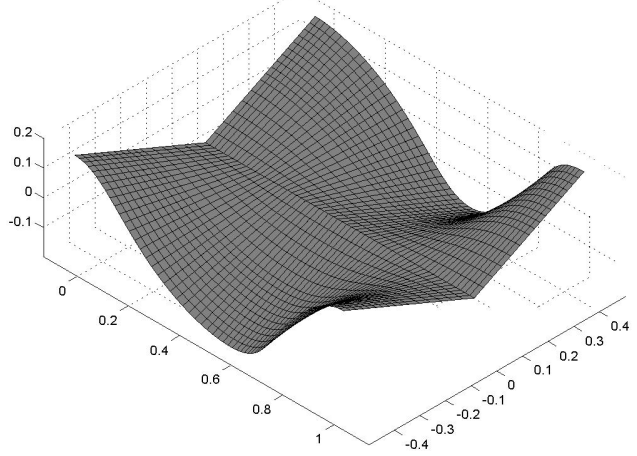

(d) Perspective View.

Figure 5. An illustration of the optimal minimum power wake shape corresponding to forward aft-flapping motions for $C_{T}=0.01$. Flight is from left to right. 


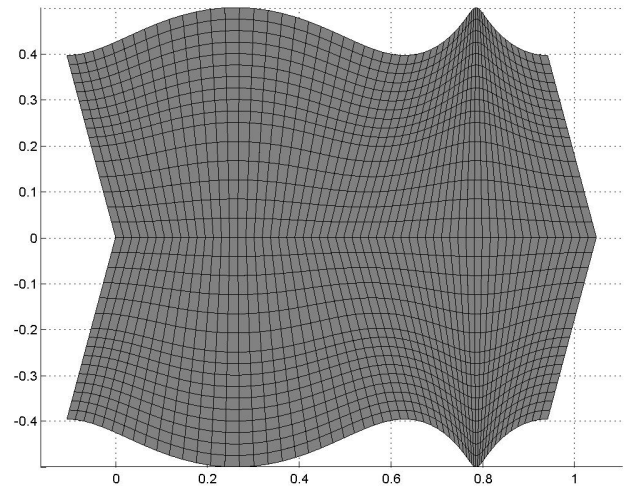

(a) Top View.

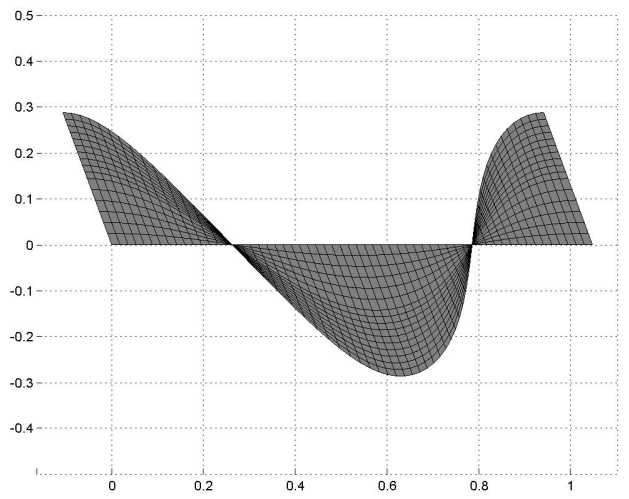

(c) Side View.

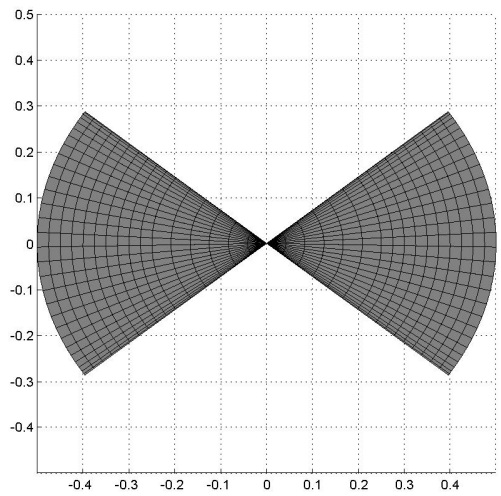

(b) Front View.

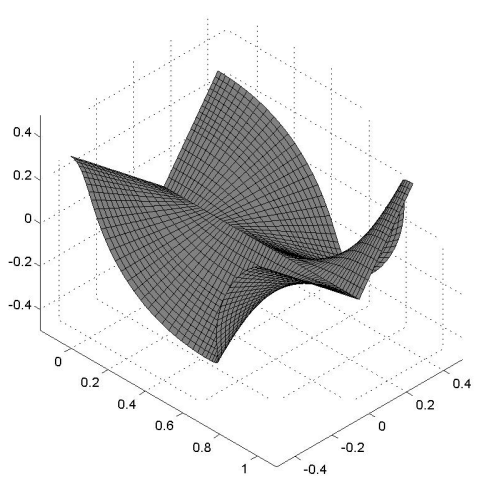

(d) Perspective View.

Figure 6. An illustration of the optimal minimum power wake shape corresponding to forward aft-flapping motions for $C_{T}=0.04$. Flight is from left to right. 


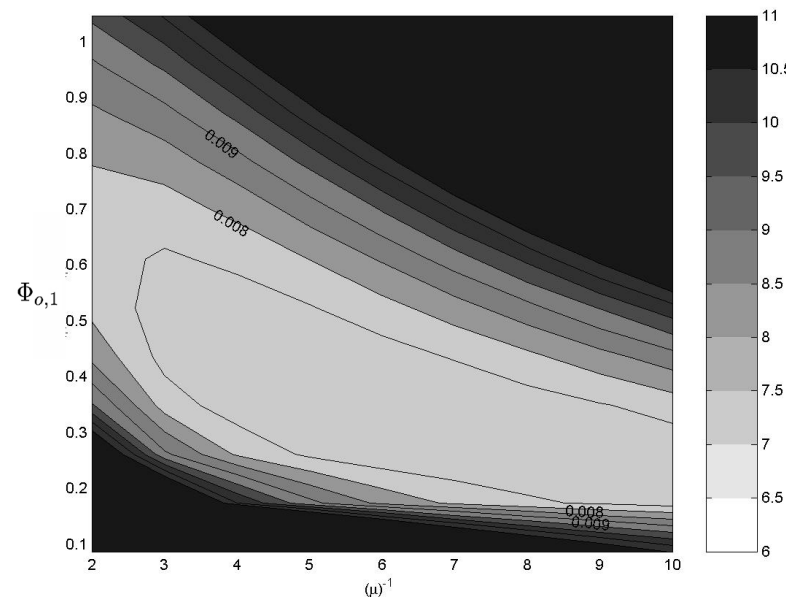

(a) Baseline, $C_{T i}=0.01$.

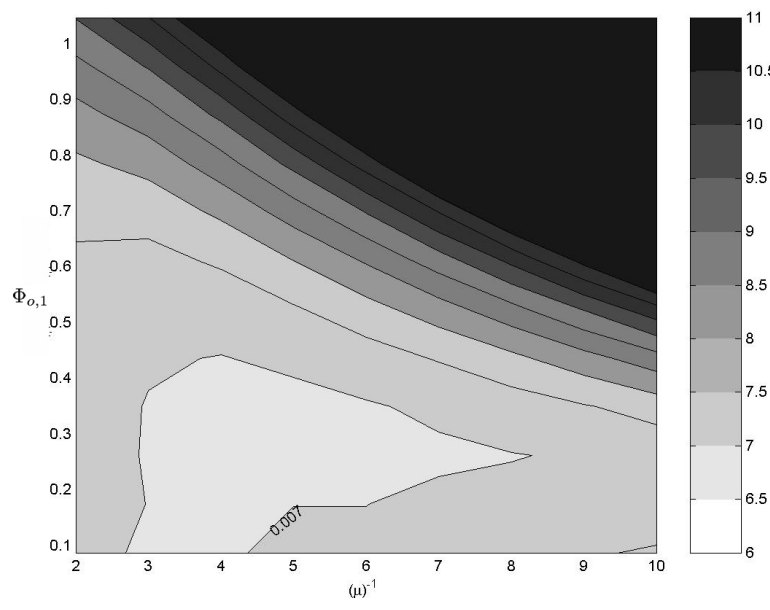

(c) Second Harmonic, $C_{T i}=0.01$.

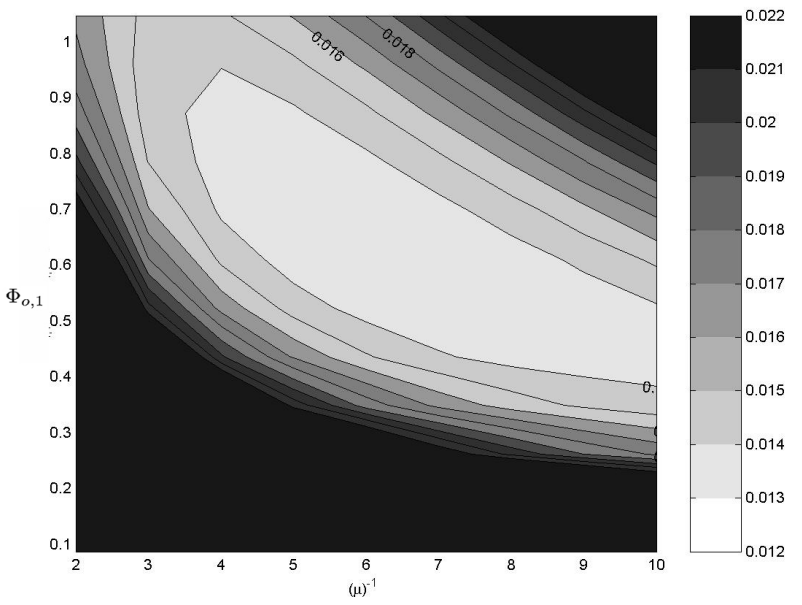

(b) Baseline, $C_{T i}=0.04$.

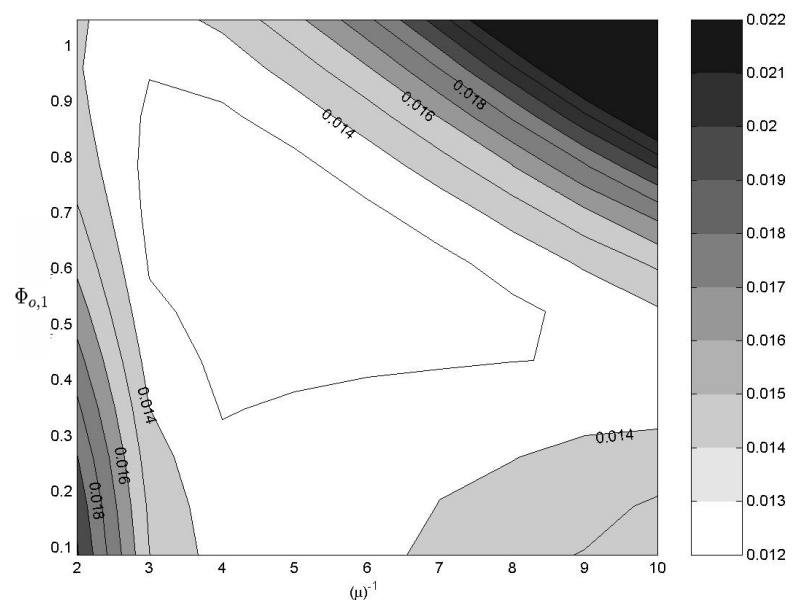

(d) Second Harmonic, $C_{T i}=0.04$

Figure 7. An illustration of the minimum power at a given root up-down flapping amplitude and advance ratio for the Baseline (simple up-down flapping motion) with one which has a second harmonic included in the wing kinematics model. 


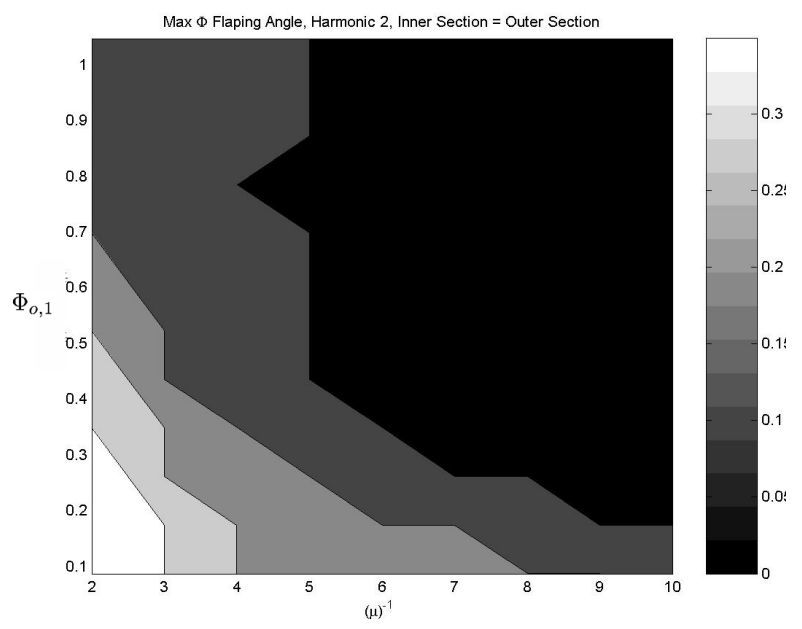

(a) $\Phi$ Amplitude, $C_{T}=0.01$

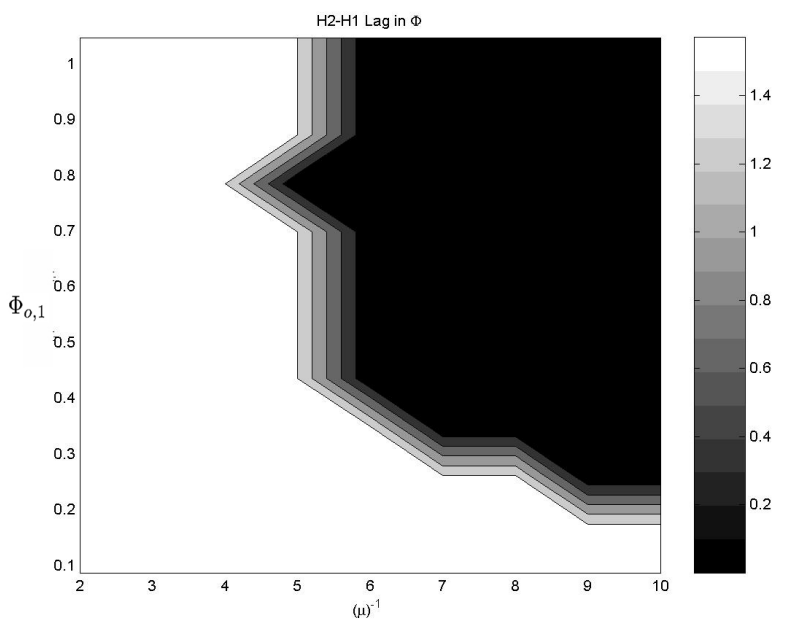

(c) $\phi, C_{T}=0.01$

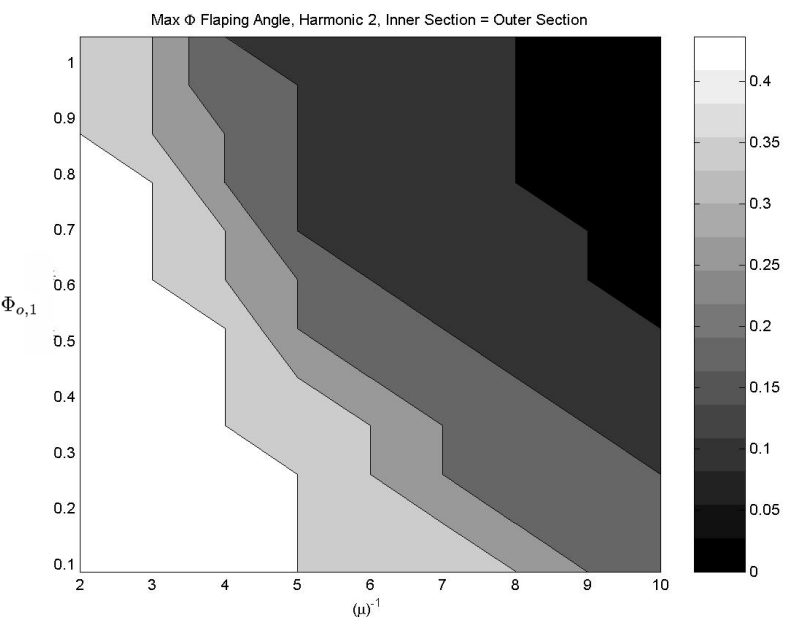

(b) $\Phi$ Amplitude, $C_{T}=0.04$

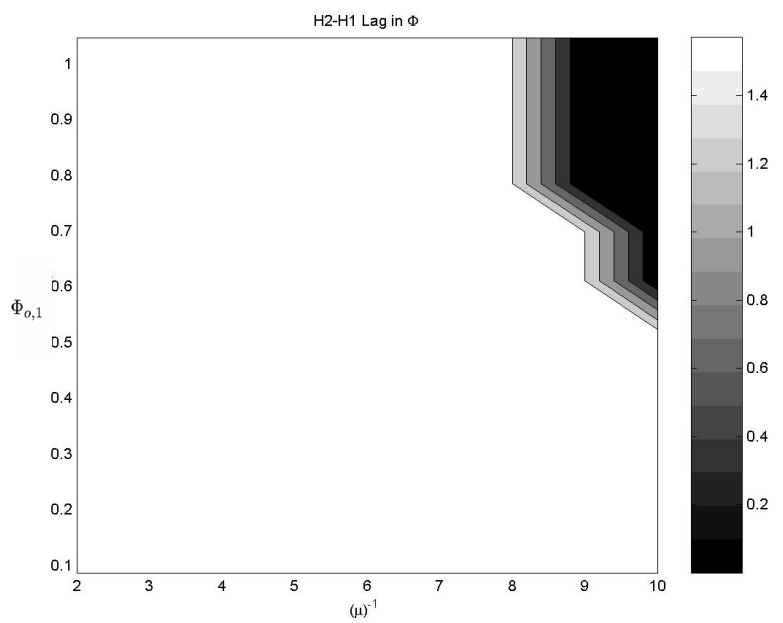

(d) $\phi, C_{T}=0.01$

Figure 8. An examination of the second harmonic flapping parameters which yield the minimum power at a given root up-down flapping amplitude and advance ratio. Note: The apparent discrete representation of the parameters is due to the coarseness of the design space investigation. 


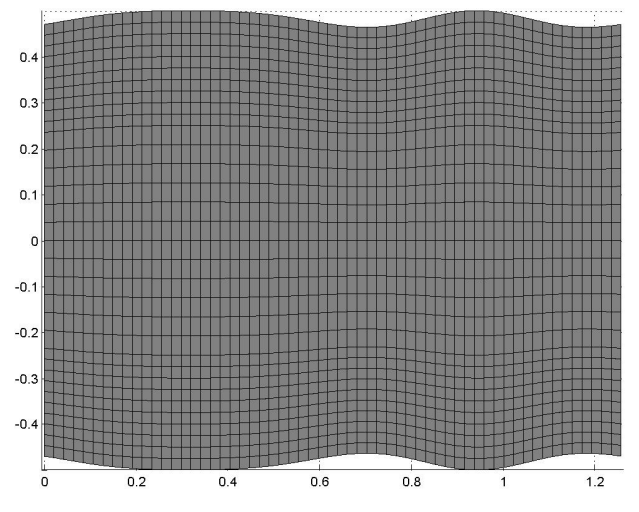

(a) Top View.

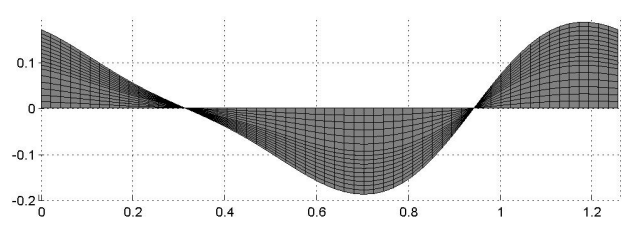

(c) Side View.

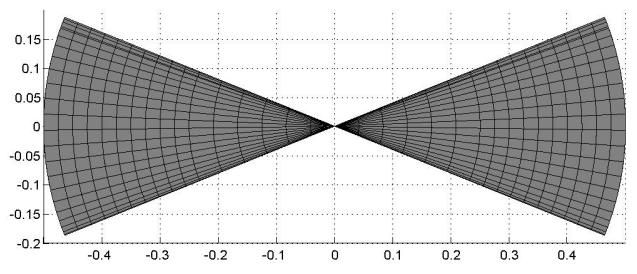

(b) Front View.

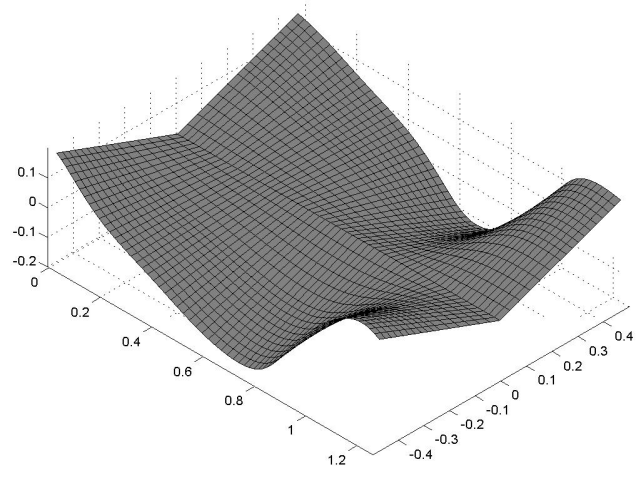

(d) Perspective View.

Figure 9. An illustration of the optimal minimum power wake shape corresponding to second harmonic motionss for $C_{T}=0.01$. Flight is from left to right. 


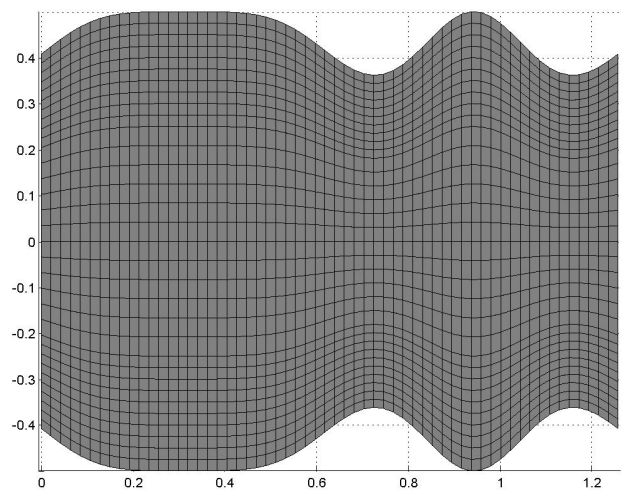

(a) Top View.

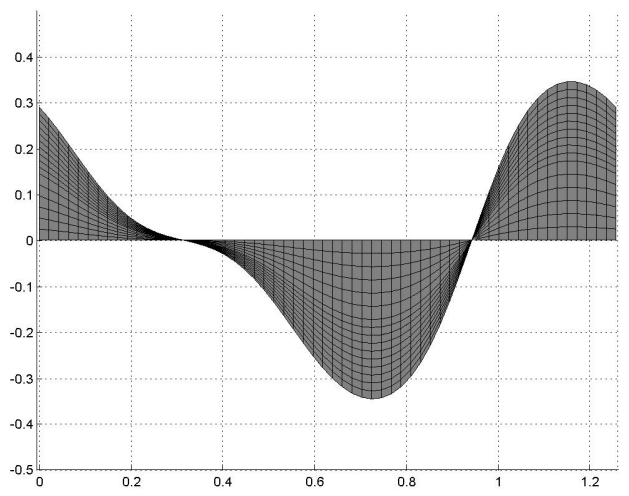

(c) Side View.

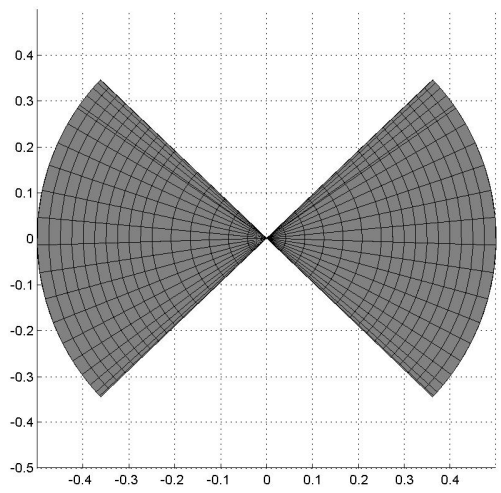

(b) Front View.

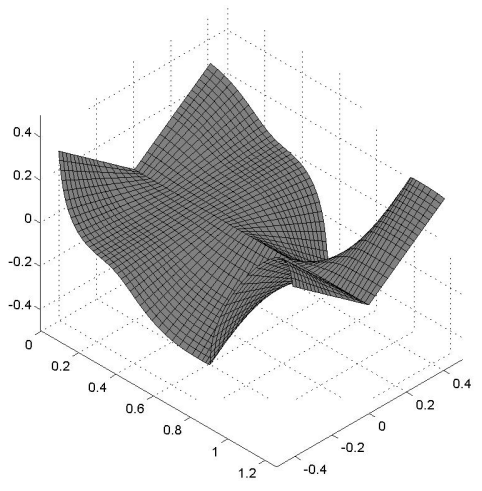

(d) Perspective View.

Figure 10. An illustration of the optimal minimum power wake shape corresponding to second harmonic motions for $C_{T}=0.04$. Flight is from left to right. 


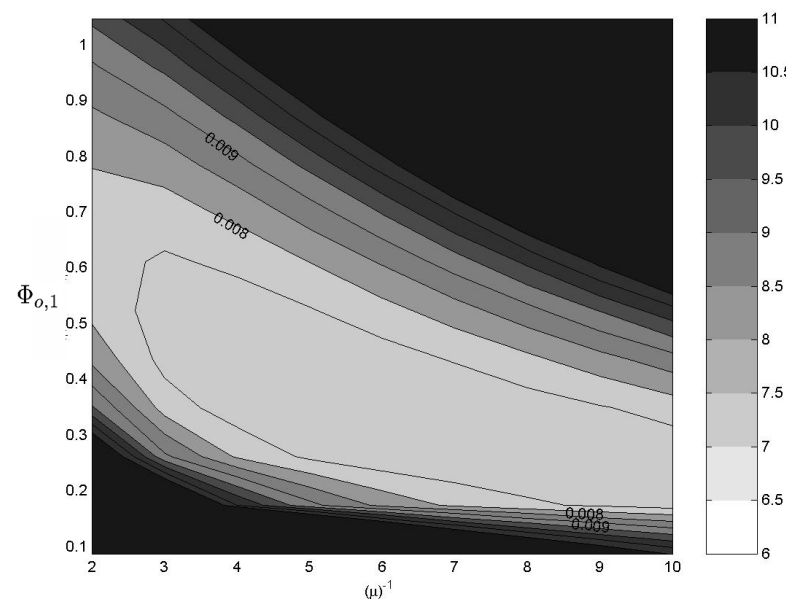

(a) Baseline, $C_{T}=0.01$.

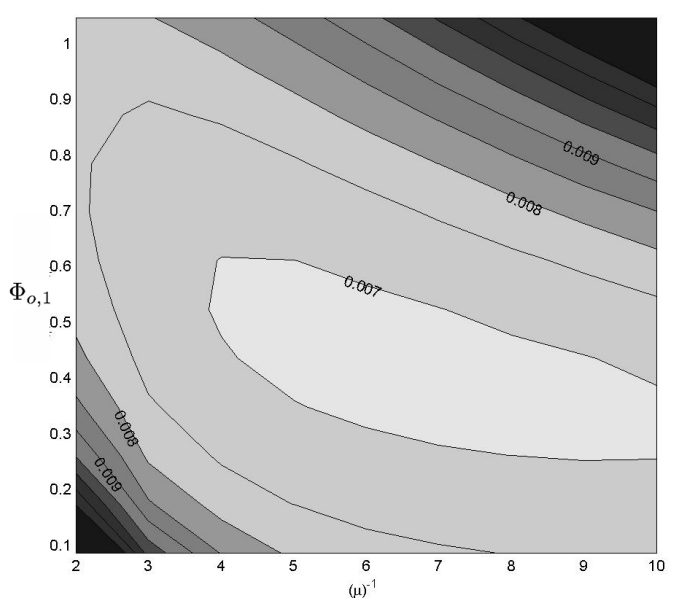

(c) Articulation, $C_{T}=0.01$.

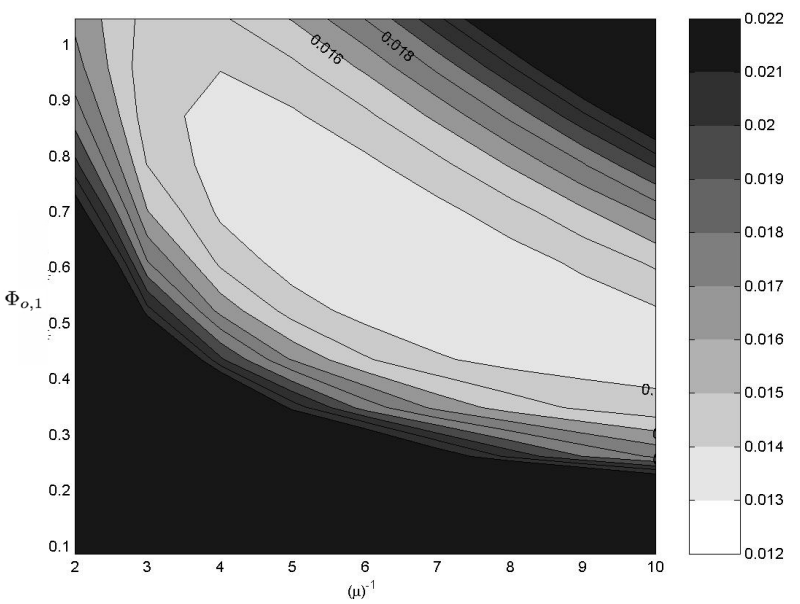

(b) Baseline, $C_{T}=0.04$.

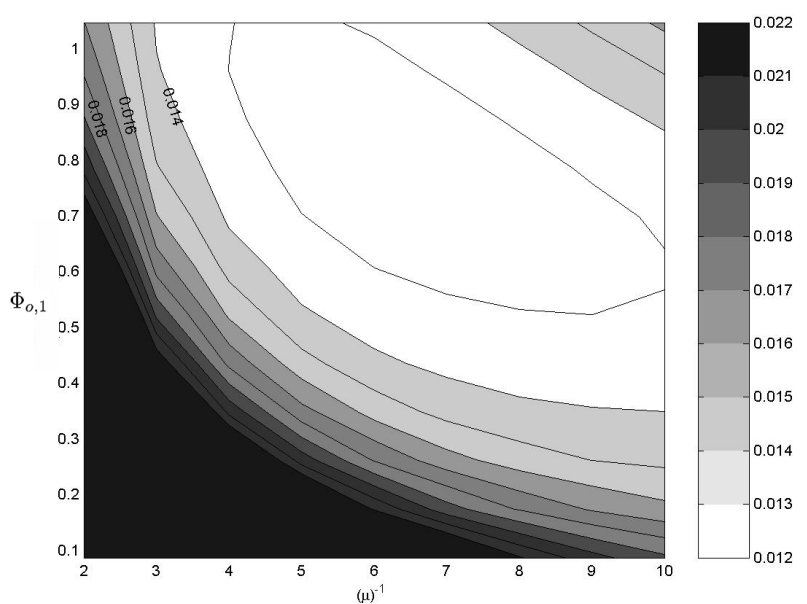

(d) Articulation, $C_{T}=0.01$.

Figure 11. An illustration of the minimum power at a given root up-down flapping amplitude and advance ratio for the Baseline (simple up-down flapping motion) with one which has a wing articulation at the wing quarter-span. 


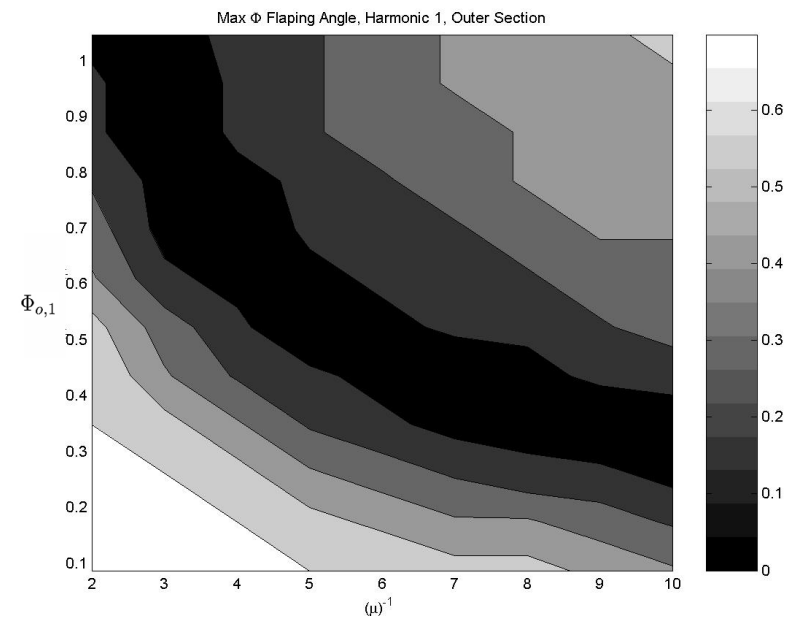

(a) $\Phi, C_{T}=0.01$.

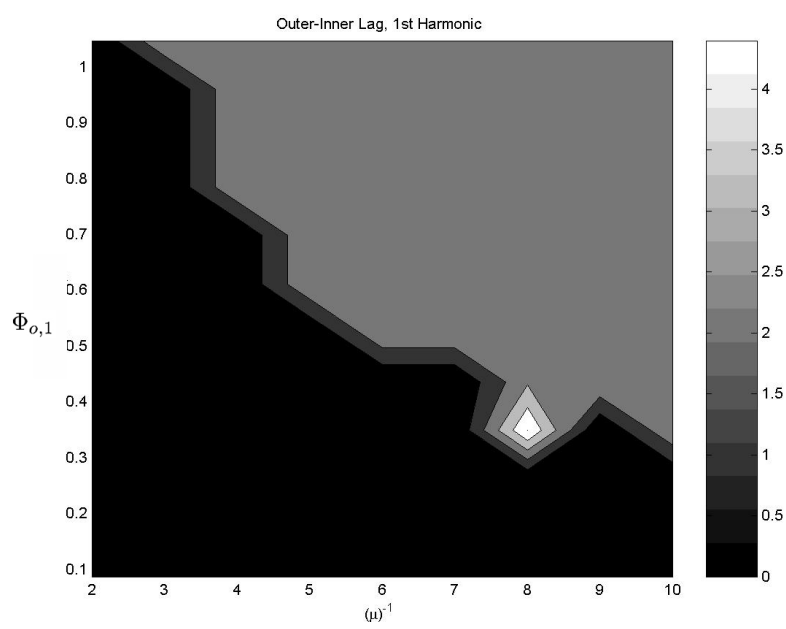

(c) $\phi, C_{T}=0.01$.

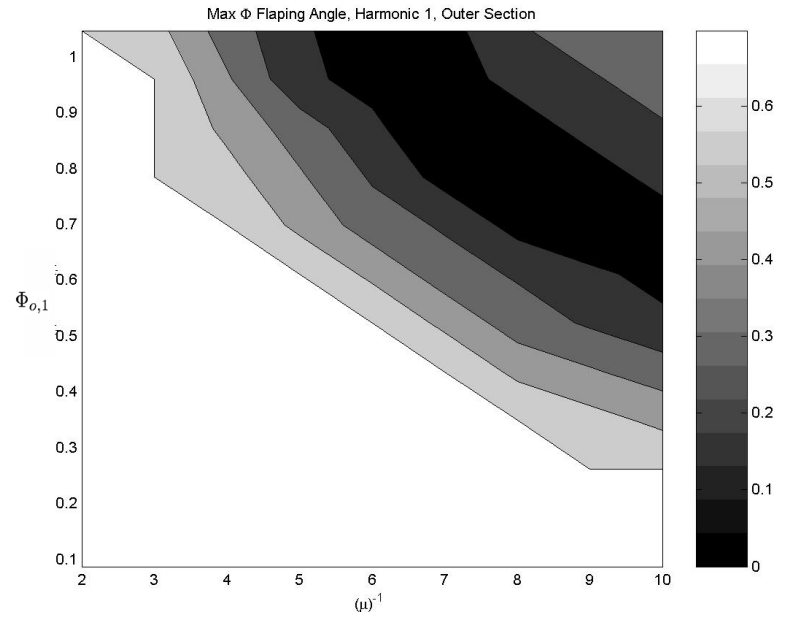

(b) $\Phi, C_{T}=0.04$.

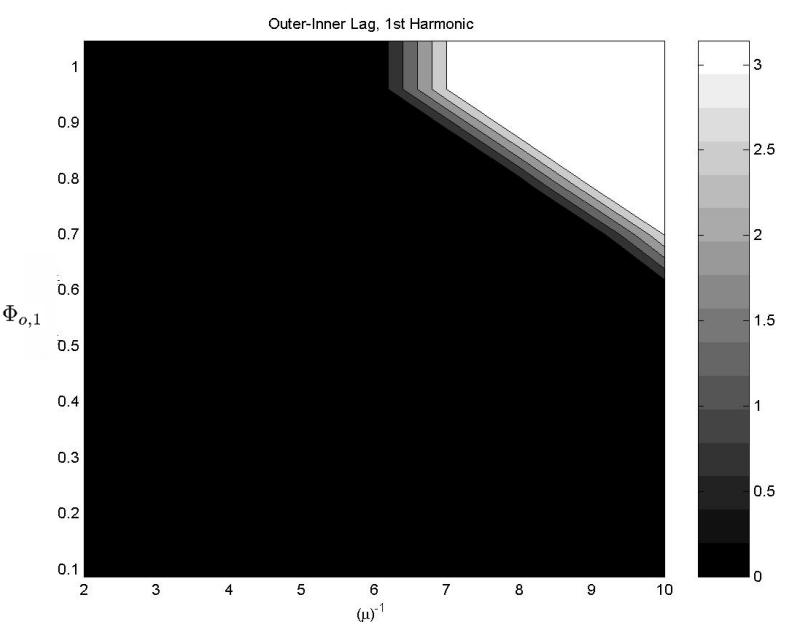

(d) $\phi, C_{T}=0.01$.

Figure 12. An examination of the articulation flapping parameters which yield the minimum power at a given root up-down flapping amplitude and advance ratio. 


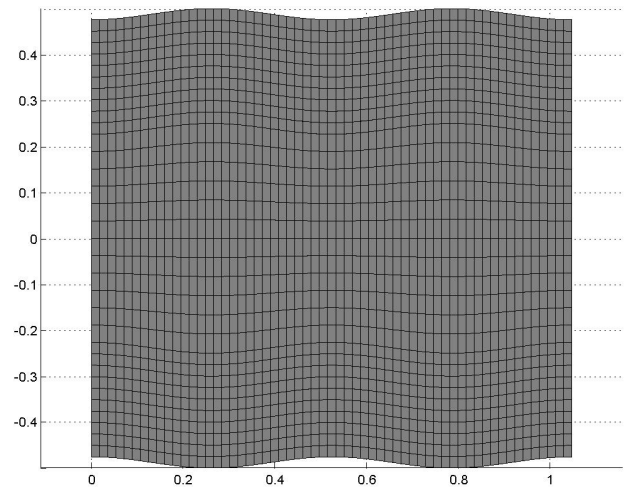

(a) Top View.

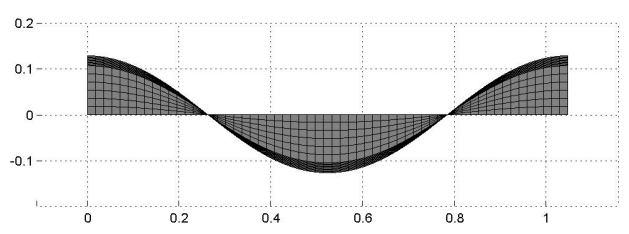

(c) Side View.

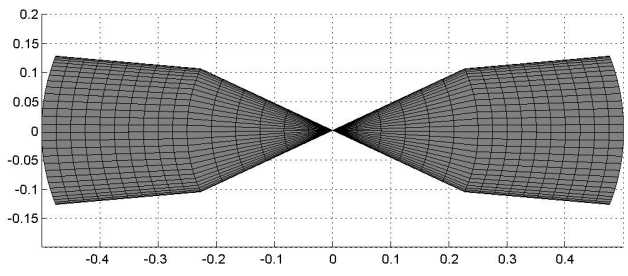

(b) Front View.

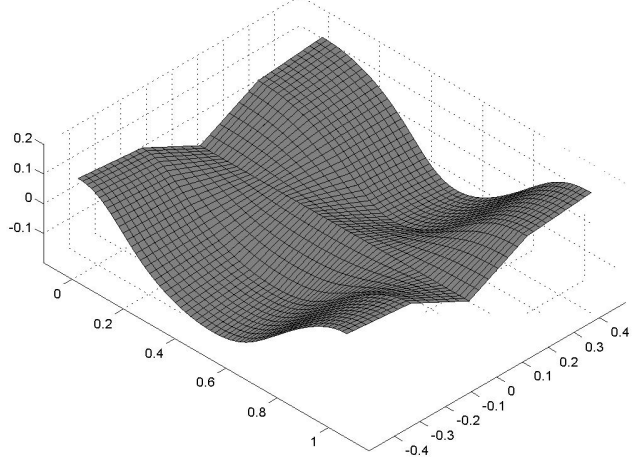

(d) Perspective View.

Figure 13. An illustration of the optimal minimum power wake shape corresponding to a quarter span wing articulation for $C_{T}=0.01$. Flight is from left to right. 


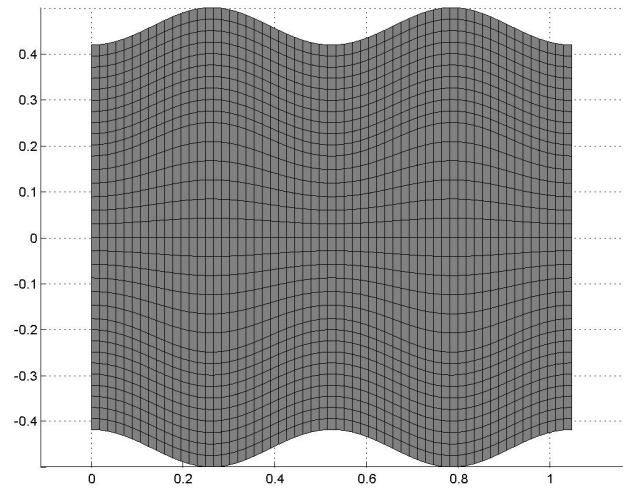

(a) Top View.

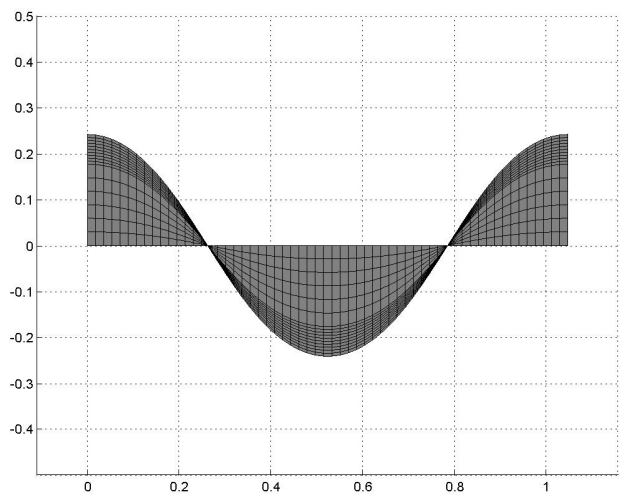

(c) Side View.

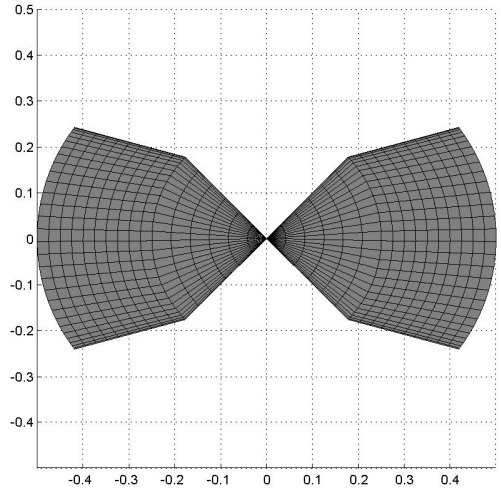

(b) Front View.

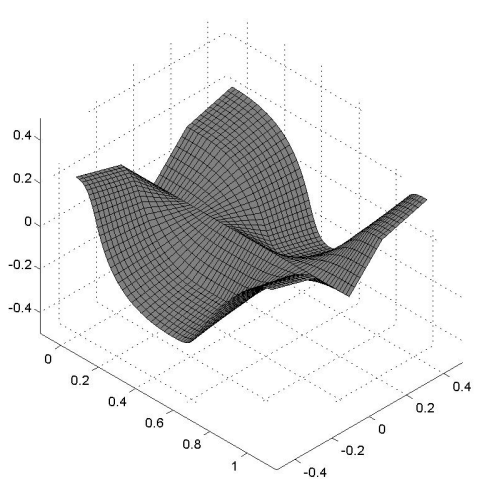

(d) Perspective View.

Figure 14. An illustration of the optimal minimum power wake shape corresponding to a quarter span articulation for $C_{T}=0.04$. Flight is from left to right. 


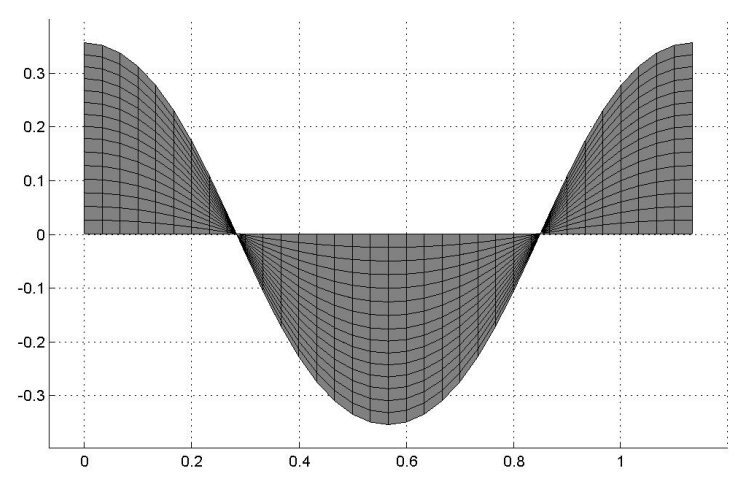

(a) Case 1r.

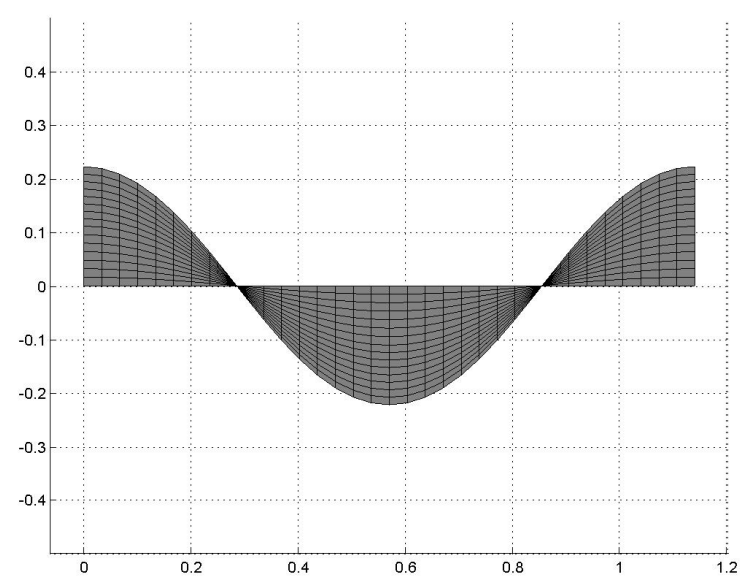

(c) case 4r.

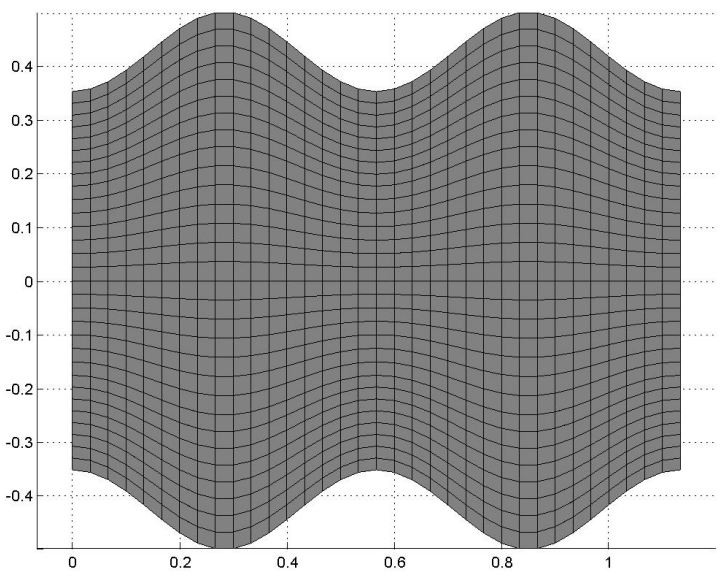

(b) Case 1r.

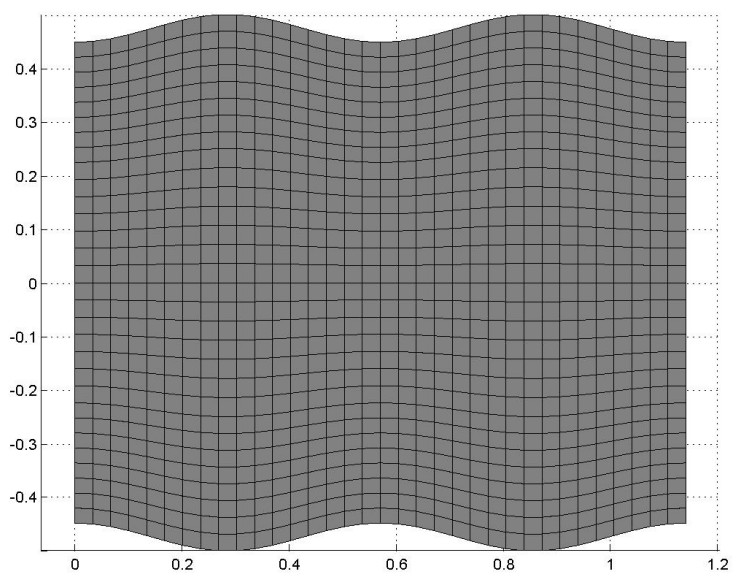

(d) Case 4r.

Figure 15. Baseline optimal wake shape. Only the wake shapes from the $C_{d 0}=0.01$ cases are shown. Flight direction is from left to right. 


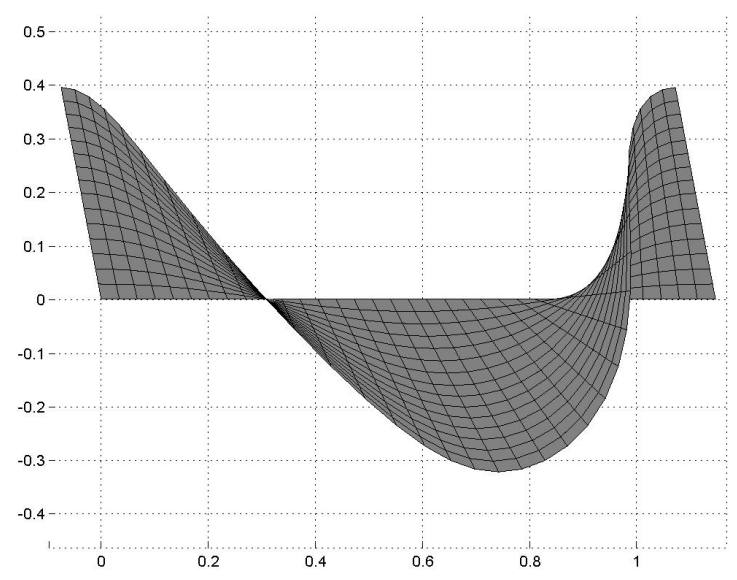

(a) Case 1a.

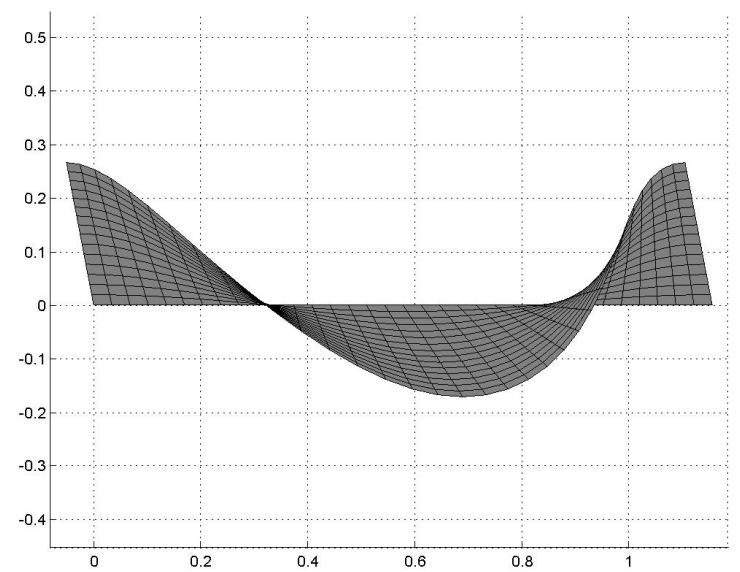

(c) Case 4a.

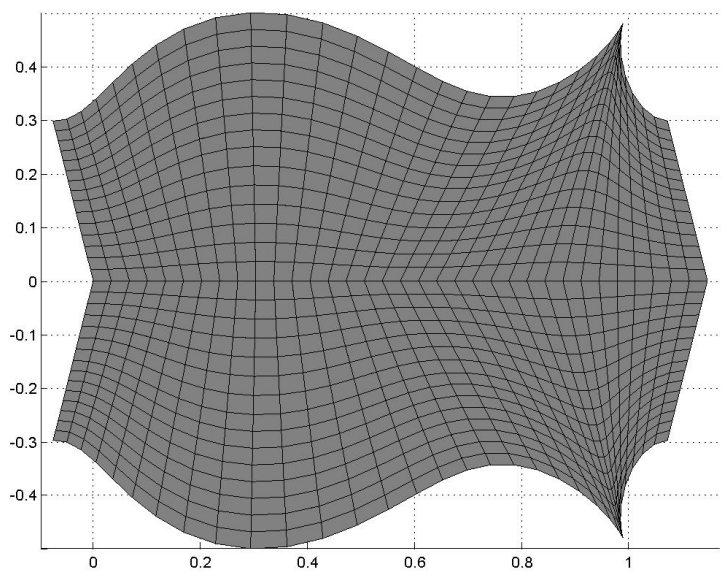

(b) Case 1a.

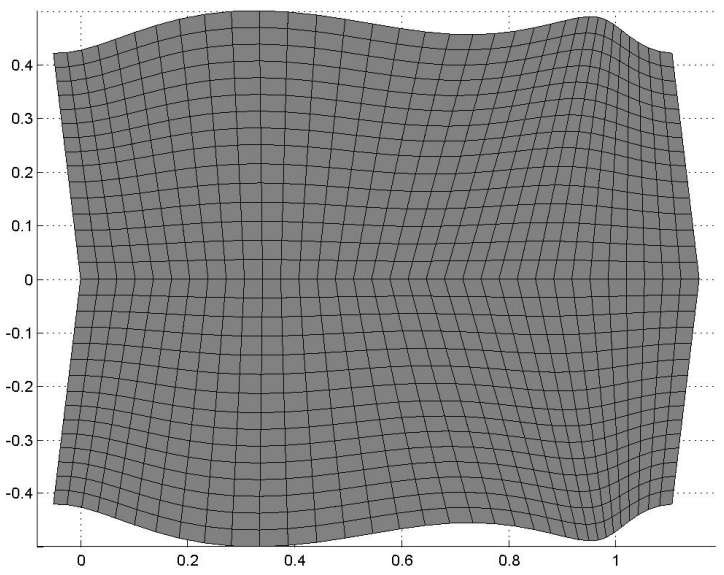

(d) Case 4a.

Figure 16. Resulting wake shape based on a forward-aft parameter freedom. Only the wake shapes from the $C_{d 0}=0.01$ cases are shown. Flight direction is from left to right. 


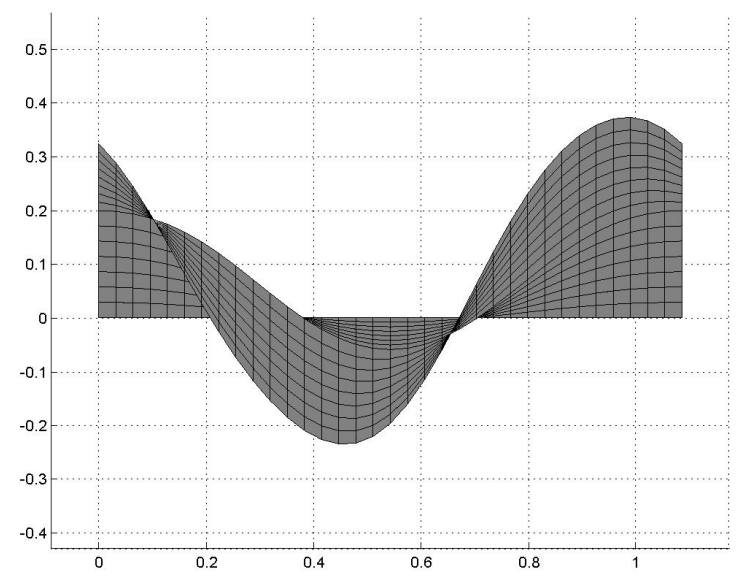

(a) Case 1b.

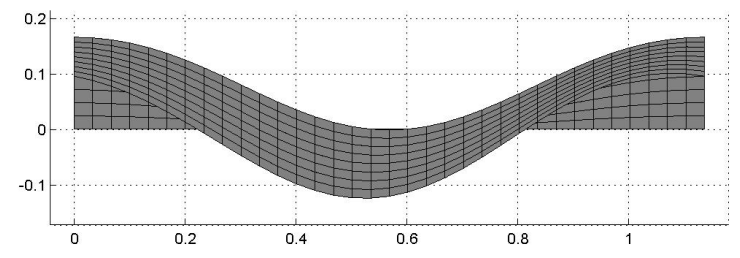

(c) Case 4b.

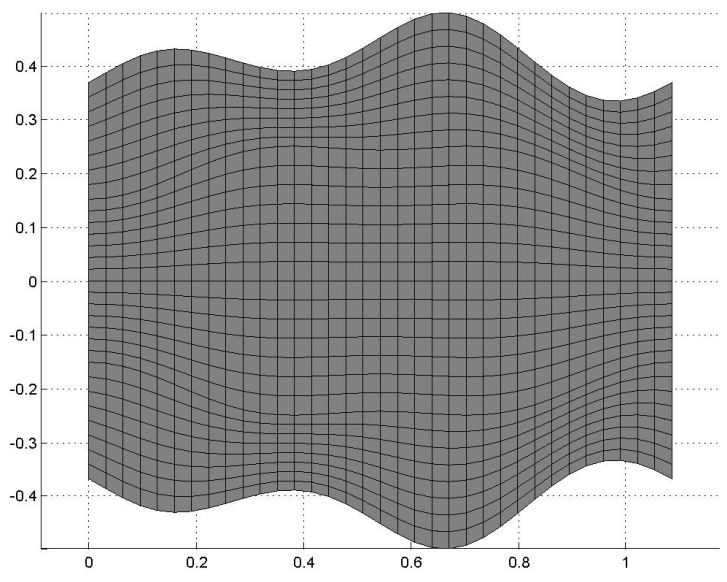

(b) Case 1b.

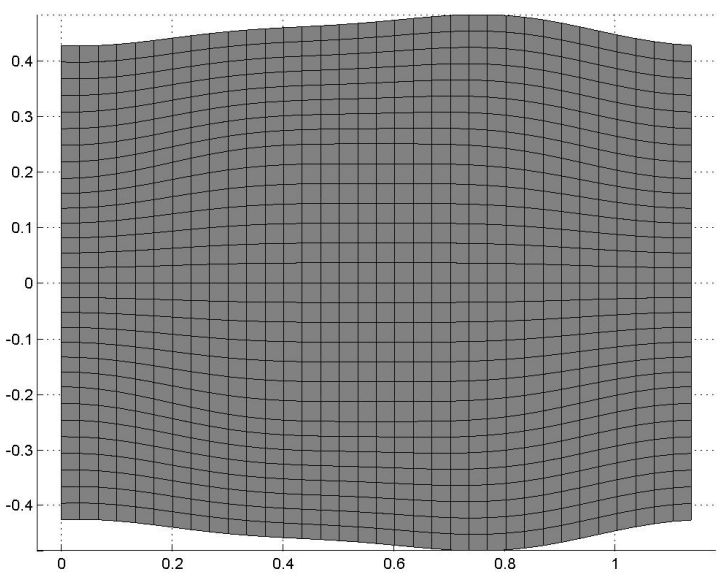

(d) Case 4b.

Figure 17. The resulting optimal wake shapes for articulation degree of freedom for the different parameter searches. Only the wake shapes from the $C_{d 0}=0.01$ cases are shown. Flight direction is from left to right. 


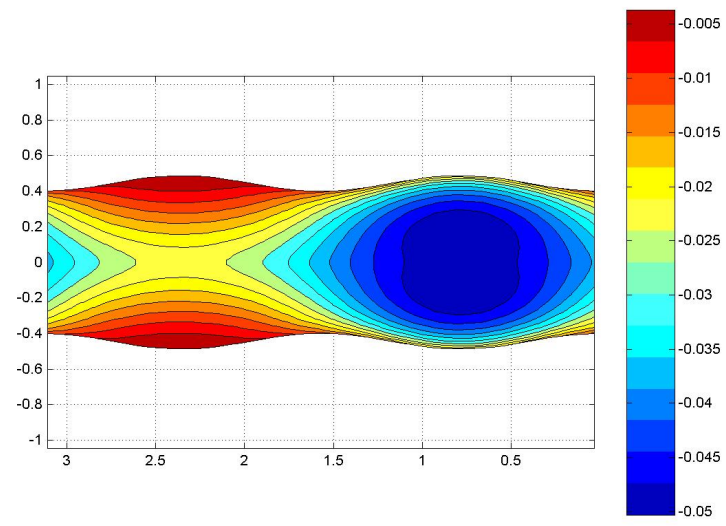

(a) The reference wake from the wake only minimum power vorticity solution. Flight path is from right to left.

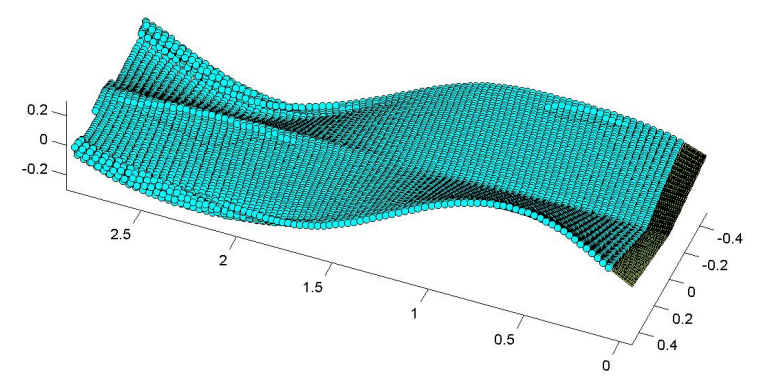

(c) An illustration of the vortex particle wake trailing a flapping wing, simulated using FastAero.

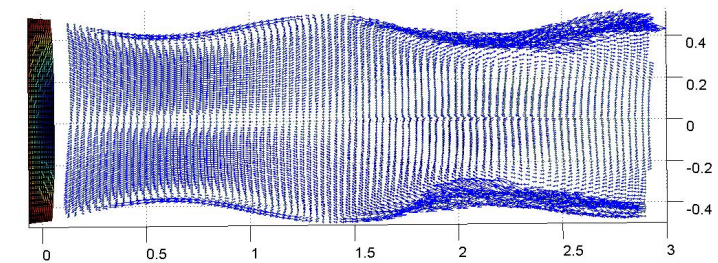

(b) A top view of the resulting FastAero simulation of a flapping geometry. Flight path is from right to left.

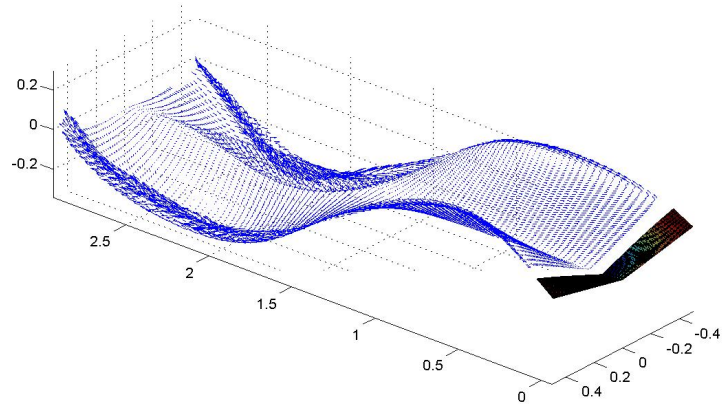

(d) An illustration of the vorticity in the wake trailing a flapping wing, simulated using FastAero.

Figure 18. A demonstration of the use of a wake only optimal vorticity distribution result to construct an efficient three-dimensional flapping wing geometry for simulation in an unsteady panel method solver. 